\title{
Microstructural Characterization of Hard Ceramics
}

Rohrer, G. S. (2014). Microstructural Characterization of Hard Ceramics. In V.K. Sarin (Editor-in-Chief) \& L. Llanes \& D. Mari (Vol.Eds.), Comprehensive Hard

Materials (pp. 265-284). Elsevier.

Gregory S. Rohrer

Department of Materials Science and Engineering

Carnegie Mellon University

5000 Forbes Ave.

Pittsburgh PA 15213-3890

phone: 4122682696

fax: 4122687596

email: gr20@andrew.cmu.edu

\section{Outline}

1. Introduction

2. Microstructural parameters

2.1. Grain sizes and Grain Size distributions

2.2. Crystal Shape

2.3. Contiguity

2.4. Crystallographic distributions of orientations and interfaces

2.5. Interface topology

2.6 Curvature Distributions

3. Recent Advances in Microstructural Characterization Techniques

3.1. Three-dimensional atom probe

3.2. Electron tomography

3.3. X-ray tomography

3.4. DB-FIB/EBSD

3.5. X-ray diffraction microscopy 
4. Summary and Conclusions

\title{
Keywords
}

Atom probe, Composite, Contiguity, Crystal shape, Electron Backscatter Diffraction, Grain boundary, Grain boundary character distribution, Grain size, Microstructure, Polycrystalline, Texture, Tomography, Topology, X-ray Microscopy

\author{
Abstract \\ This chapter reviews recent developments in the microstructural characterization \\ of hard ceramics. It begins with a survey of the most important microstuctural \\ features that can be derived from characterization experiments. These include grain \\ size, grain shape, contiguity, texture, the grain boundary character distribution, and \\ curvature. In each case, the parameters are illustrated by examples of data. The \\ remainder of the chapter focuses on recent advances in three-dimensional \\ microstructural characterization. Examples of progress in the areas of three- \\ dimensional atom probe, electron and X-ray tomography, dual beam FIB analysis, \\ and X-ray diffraction microscopy are all discussed.
}




\section{Introduction}

The study of microstructures is motivated by the fact that nearly all engineered materials are used in a polycrystalline form. The realization that many of the macroscopic properties of polycrystalline solids are strongly influenced by the internal structure of the polycrystal has motivated countless microstructure studies, most with the goal of establishing correlations between the microstructure and the properties.

This chapter will focus on the most recent developments in microstructural characterization and their applications to ceramics. However, at the outset, it is appropriate to note past reviews of this topic. For example, the relationship between carbide (Hollox, 1968, Tucker and McLachlan, 1993) and carbide composite microstructures (French, 1969, Gurland, 1988, Sherman and Brandon, 1999) and mechanical properties has been thoroughly reviewed. The application of electron microscopy to ceramic microstructure (Bando, 1995, Kleebe et al., 1996, Thomas, 1996) and grain boundaries (Ikuhara, 2001, Varela et al., 2005) has also been the subject of several comprehensive reviews.

While there are a few early examples of novel three-dimensional studies (Hull, 1988, Williams and Smith, 1952), the vast majority of microstructural information has been determined from the analysis of two-dimensional images. The microstructure is, of course, a three-dimensional object. Stereology, which is the interpretation of three-dimensional spatial information from two-dimensional sections, has been the link that allows volumetric information about the microstructures to be drawn from the planar images. However, all stereological 
results depend on an assumption of homogeneity and, in some cases, geometric models or assumptions about the true shapes of objects in three-dimensions. In cases where these assumptions are valid, the stereological results are also valid. (Russ, 1986) However, for many of the most interesting materials, the validity of the assumptions cannot be guaranteed.

In the past decade, there has been a rapid expansion in capabilities for threedimensional microstructure studies; this has provided an entirely new perspective on some "well-known" microstructures and pointed to the weakness of the assumptions used in stereology. As an example, one can point to a landmark study of the three-dimensional structure of cementite precipitates in a ferrous alloy which showed that structures historically classified as intragranular precipitates were actually branches connected to dendritic grain boundary precipitates and Widmanstätten structures. (Kral and Spanos, 1999) Considering the expected value of these emerging capabilities for the materials science community, this chapter will emphasize the three-dimensional characterization of microstructures.

This recent revolution in our ability to characterize microstructures in three dimensions has occurred across a wide range of length scales from the atomic to the millimeter range. (Robertson et al., 2011) At the atomic scale, there have been significant advances in field ion microscopy. Although this technique has been available for decades, the limited volume of material that could be imaged and the types of materials that could be imaged made it impractical for many studies. However, the recent development of laser assisted, three-dimensional atom probe microscopy (3DAP) has made it possible to make three-dimensional, near-atomic 
resolution images of reasonable volumes of a wide range of materials. (Kelly and Miller, 2007, Miller and Forbes, 2009, Seidman, 2007) At the same time, advances in electron tomography in the transmission electron microscope (TEM) have made it possible to make three-dimensional images at slightly lower resolution. (Barnard et al., 2006, Batenburg et al., 2009, Mobus and Inkson, 2001, Weyland et al., 2006) The Dual Beam, Focused Ion Beam Scanning Electron Microscope (DB-FIB SEM) makes three-dimensional imaging and crystallography mapping possible at the submicron level. (Dillon and Rohrer, 2009b, Groeber et al., 2006, Li et al., 2009, Rohrer et al., 2010, Uchic et al., 2006) At larger length scales, X-ray tomography (Chen et al., 2010, Chino and Dunand, 2008) and high energy X-ray diffraction microscopy (HEDM) (Hefferan et al., 2009, Johnson et al., 2008, Larson et al., 2002, Poulsen et al., 2001) allow phase mapping and crystallographic mapping, respectively. Finally, at the largest length scale, "RoboMet" robotic polishing and metallography allows the three-dimensional imaging of samples with sizes up to the $\mathrm{cm}$ size range. (Spowart et al., 2003)

In microstructure characterization, there is always a compromise between resolution and the number of features (for example, grains) that can be observed and the limits depend on the instruments used. This is especially true in threedimensional studies. The compromises between resolution and the numbers of features is summarized by the schematic in Fig. 1. (Rohrer, 2011b) Using current techniques and those anticipated in the near future, the approximate bounds on domains are shown by the solid lines; all of these domains are expected to exapand to the right with time. These techniques make it possible to create input for 
computational models that can be used to predict materials properties. One key aspect of Fig. 1 is that, depending on the problem at hand, it may not be possible to characterize all of the relevant length scales without using a combination of techniques.

$<$ Figure 1 near here $>$

Simulation and modeling are playing an increasingly important role in establishing structure-property relationships and there is an emerging vision of being able to rapidly develop and optimize new materials through an increased interplay between simulation and experiment. (2011, Pollock et al., 2008) Realizing this vision, however, requires accurate, three-dimensional, microstructural data. These trends provide a renewed motivation for improved characterization and analysis techniques.

The remainder of this chapter is organized in the following way. First, the most important microstuctural features that one can derive from characterization experiments are described and illustrated by some examples of data. Next, the most important recent advances in three-dimensional microstructural characterization are described. Although examples from a wide range of materials types will be used, all of the methods are applicable to ceramics and other hard materials. Finally, a summary and prognosis for future trends are provided. 


\section{Microstructural parameters}

\subsection{Grain sizes and Grain Size distributions}

As mentioned in the introduction, most materials are used in a granular form and are either polycrystalline (in the case of single phase materials) or are composites consisting of two or more phases. In all cases, the sizes of the component crystals are an important characteristic of the microstructure that is connected to physical properties including strength, hardness, and optical transparency, to name a few. From planar sections, the crystal size is usually measured using the linear intercept method. Using this method, the number of intercepts per length of test lines that are superimposed on the microstructure is used as a measure of the grain size. For example, for the image in Fig. 2(a) of a WC/Co composite, a skeleton (see Fig. 2(b)) can be created that consists of all of the carbide-carbide and carbide-binder interfaces, where Co is the binder. It is then relatively simple to count the number of times a line of fixed length intercepts each boundary. (Kim et al., 2008) The stereologically determined carbide grain size, $L^{*}$, is a function of the volume fraction of the carbide $\left(f_{c}\right)$ and the average number of intercepts per unit length of test lines with carbide/carbide boundaries $\left(N_{c c}\right)$ and binder/carbide interfaces $\left(N_{b c}\right)$ :

$$
L^{*}=\frac{2 f_{c}}{2 N_{c c}+N_{b c}}
$$

$<$ Figure 2 near here $>$ 
In the case that three-dimensional data is available, there is no need for a stereological interpretation and it is possible to determine grain sizes directly in terms of either their volume or spherical equivalent radii. (Groeber et al., 2006, Rowenhorst et al., 2010) The distribution of crystal sizes is most frequently reported to be lognormal (Rohrer, 2005):

$$
f_{L}(\rho)=\frac{1}{(2 \pi)^{1 / 2}} \frac{1}{\beta \rho} \exp \left[-(\ln \rho-\alpha)^{2} / 2 \beta^{2}\right]
$$

where $\rho=r /<r>,<r>$ is the average grain size, and $\alpha$ and $\beta$ are fitting parameters. On a coarse scale, the lognormal distribution provides a good account of distribution. For example, the data shown in Fig. 3 for WC crystal sizes in a WC-Co composites demonstrates that in many different samples, the width of the distribution is the same and only the mean varies. (Kim et al., 2008) However, there can be significant deviations in the tails of the distribution that are not obvious in the plots such as Fig. 3(a). For example, Fig. 4 shows that the lognormal distribution is accurate for features that are within one standard deviation of the mean, but that there are more large grains than predicted by the lognormal distribution. (Tucker et al., 2012)

$<$ Figure 3 near here $>$ $<$ Figure 4 near here $>$ 
While knowledge of the features close to the mean is sufficient for determining average materials properties, such as elastic response or thermal conductivity, it is the rare features that frequently trigger dramatic changes in the microstructure, often with life-limiting consequences for applications. For example, recent simulations have shown that the long-term capacity of a rechargeable Li-ion battery depends on the extremes of the grain size distribution in the anode and cathode and that Li dendrite formation, which leads to unpredictable battery behavior, occurs on the smallest grains. (Smith et al., 2009) Similarly, when the size distribution of grains that initiate fatigue cracks in a Ti alloy are compared to the overall size distribution, it is found that the initiating grains are larger than the mean. (Jha et al., 2009)

\subsection{Crystal Shape}

Grain shapes are most frequently interpreted in terms of some model geometric shape, such as a circle (sphere), ellipse (ellipsoid), or polygon (polyhedron) in two dimensions (three dimensions). For example, the transverse section of the CVDgrown $\mathrm{TiC}_{\mathrm{x}} \mathrm{N}_{1-\mathrm{x}}$ coating shown in Fig. 5 has grains elongated along the growth direction. (Chien et al., 2009) Freely available software (Health) can be used to fit the grain shapes in the image to ideal ellipses. From this, it is possible to determine the average area and the average dimensions of the major and minor axes. For the materials shown in Fig. 5, the ratio of the major to minor axis length is 7.2.

$<$ Figure 5 near here $>$ 
It is also possible to approximate crystals as polyhedra. However, in this case it is also necessary to have a measure of crystal orientation, so electron backscatter diffraction (EBSD) data is necessary. A stereological technique has been developed to calculate the relative areas of different types of interfaces based on twodimensional images. (Saylor et al., 2004b, Saylor and Rohrer, 2002) The required observations for the stereological analysis are line segments, in the sample reference frame, representing the crystal boundaries (see Fig. 6(a)), and the associated crystal orientations. From these data, the interface plane distribution (IPD), which is the relative areas of crystal planes as a function of orientation, can be calculated using freely available software. The IPD, $\lambda(\mathbf{n})$, can be measured for the grain boundaries in a single phase material or the phase boundaries and grain boundaries in a composite.(Kim et al., 2008, Kim and Rohrer, 2004, Sano et al., 2005) The IPD has two parameters that specify the interface normal orientation and can be plotted as a continuous distribution, as in Fig. 6(b).

$<$ Figure 6 near here $>$

For the case of regular faceted system, such as WC crystals in a Co matrix, the crystal shape can be modeled as a trigonal prism with $\{0001\}$ (Syha et al., 2012) basal facets and (10 $\overline{1} 0)$ prismatic facets (see Fig. 6(c)). (Kim et al., 2008, Kim and Rohrer, 2004) In this case, the shape can be parameterized by an aspect ratio computed as the ratio of the length along the edge of the (0001) basal facet (b) of its height (h) along a (1010) facet. The results in Fig. 6(c) show how the WC aspect 
ratio varies with the volume fraction of $\mathrm{WC}$ and Co. In a stereological determination of crystal shape, it is necessary to have a sufficient number of observations. To illustrate this, Fig. 7 shows the aspect ratio of WC crystals calculated using different numbers of observations. Based on these results, it is clear that after approximately 500 observed line segments, the result is not affected by additional data. This provides a guideline for the minimum number of line segments that are required for an accurate IPD.

$<$ Figure 7 near here $>$

If the data is three-dimensional, then no stereological interpretation is required and grain shapes can be determined directly. In this case, it is possible to specify the ranges of shapes and how they vary. This is not straightforward for complex shapes, but parameters have been developed to quantify shape characteristics and their distributions. For example, MacSleyne et al. have used moment invariants to quantify the three-dimensional shapes of precipitates in microstructures.

(MacSleyne et al., 2009) Moment invariants are combinations of the moments of inertia of an object that are invariant with respect to certain transformations. For example, Fig. 8 shows how the shapes of dendritically shaped precipitates can be classified using moment invariants. The vertical axis is an affine parameter that is invariant with respect to translations, rotations, anisotropic scaling, and shear. The horizontal axis is a shape parameter that measures of the volume-to-surface ratio (see MacSleyne, 2009 for the detailed definitions, which are beyond the scope of this chapter). This representation clearly separates highly branched structures (lower 
left) from more compact structures (upper right). While the application of these distributions is still being studied, they offer a promising path for the quantification of crystal shapes in microstructures.

$<$ Figure 8 near here $>$

\subsection{Contiguity}

In composite materials, the contiguity of each phase can affect the mechanical properties, especially in the case where there is significant contrast in the properties. For the case of WC/Co composite, contiguity of the carbide phase $(C)$ is computed according to the following expression:

$$
C=\frac{2 \sum_{i} l_{c c}^{i}}{\sum_{i, j}\left(2 l_{c c}^{i}+l_{b c}^{j}\right)}
$$

where $l_{c c}$ is the length of a carbide-carbide grain boundary and $l_{b c}$ is the length of a binder-carbide interface. These lengths can be extracted from micrographs such as those illustrated in Figures 2(a) and 6(a). Figure 9 illustrates data for the contiguity of WC crystals in WC/Co composites as a function of the WC volume fraction. (Kim et al., 2008) While these data indicate that the contiguity is approximately linear with the carbide volume fraction over the range of compositions examined, more comprehensive studies, over a wider range of composition, have found that the contiguity increase is non-linear. (German, 1985) The data in Fig. 9 also contains evidence that the carbide volume fraction / contiguity relationship is also scale 
invariant in the range of specimens studied: samples A and B have very different grain sizes (5.31 and $1.65 \mu \mathrm{m}$, respectively), but nearly the same carbide volume fractions and contiguity. (Luyckx and Love, 2006)

$<$ Figure 9 near here $>$

Contiguity in three dimensions can be determined exactly. This is particularly important for devices such as solid oxide fuel cells where the contiguity of the void space (to transport reactant gases) and of the three phase boundaries (where gases, ionic species, and electronic charge carriers can participate in reactions) influences the overall transport through the device and, therefore, the electrochemical characteristics. Wilson et al. studied the anode of a solid oxide fuel cell comprised of $\mathrm{Ni}$, yttria stabilized zirconia, and pore space and found that of all the three phase boundaries, $63 \%$ of the length formed an interconnected network. (Wilson et al., 2006)

\subsection{Crystallographic distributions of orientations and interfaces}

The term "texture" is usually used to refer to a non-random distribution of crystal orientations. However, it can also refer to a non-random distribution of misorientations or interface planes. Orientation textures are typically quantified using pole figures (distributions of the normals to a specific set of crystal planes in the sample reference frame) and inverse pole figures (distributions specific sample directions in the reference frame of the crystals) that are calculated from either X- 
ray or electron backscatter diffraction (EBSD) data. As an example, consider the electron backscatter diffraction orientation map of $\alpha$-alumina in Fig. 10 and the accompanying inverse pole figure and pole figure. (Chien et al., 2009) This map is a transverse section of a coating grown by chemical vapor deposition, so the growth direction is vertically oriented in the plane of the figure. The dominance of blue and green colors in Fig. 10(a) indicates that the prismatic orientations (those perpendicular to the [0001] axis) are perpendicular to the plane of the image and the [0001] axis is aligned along the growth direction. This is also reflected in the inverse pole figure (IPF) in Fig. 10(b) and the pole figure in 10(c). The IPF shows the distribution of crystal orientations along the growth direction of the coating. It is consistent with the conclusions from the color in the orientation map, but quantifies the preference for the alignment of the [0001] axis along the growth direction. The units, multiples of a random distribution (MRD), compare the actual distribution to the distribution expected for the case when the orientations are completely random. If the value is less than one, this indicates that the orientation occurs less frequently than one would expect in a random distribution. If it is greater than one, it occurs more frequently by that multiple. This is a common unit of measure in texture studies. The pole figure in Fig. 10(c) also shows that the [0001] axes are preferentially aligned with the growth direction.

$<$ Figure 10 near here $>$ 
Interfaces and grain boundaries can also exhibit texture. In the most general sense, the relative areas of different types of grain boundaries are described by the grain boundary character distribution (GBCD). (Rohrer et al., 2004) The grain boundary type is specified by five parameters. As parameters for this distribution, we select a misorientation type by specifying an axis-angle combination (three parameters), and a grain boundary plane orientation by specifying a unit vector normal to the boundary (two parameters). It is possible to characterize the GBCD using fewer than five parameters and a variety of representations are illustrated in Fig. 11 and discussed in the nest paragraph. (Kim et al., 2008, Rohrer, 2011b)

$<$ Figure 11 near here $>$

Figure 11 shows three different representations of the GBCD calculated using the same data. This example is for hexagonal WC. Figure 11(a) shows a single parameter distribution of disorientations, in which each boundary is classified by its minimum misorientation angle, without consideration of the misorientation axis. Compared to the random distribution, there is an enhancement of grain boundaries with $30^{\circ}$ and $90^{\circ}$ disorientations. When one considers the axis and angle of the misorientation, there are three independent parameters for each boundary, two for the axis direction and one for the rotation angle. Therefore, in Fig. 11(b) each position in a three-dimensional space corresponds to a different grain boundary misorientation. In each layer of the axis-angle space, all possible axes are represented; the rotation angle varies along the vertical direction. Note that the peak for $30^{\circ}$ in $11(a)$ is concentrated at [0001], indicating that these are mostly $30^{\circ}$ 
rotations about [0001], and the peak at $90^{\circ}$ in $11(\mathrm{a})$ is concentrated at [10-10], indicating that this is the dominant misorientation axis. For any particular axisangle combination, there is a distribution of grain boundary planes, as shown for the examples in 11(c) and 11(d). Note that when the misorientation axis, the misorientation angle, and the grain boundary plane are specified, there are five independent parameters. The grain boundary plane distributions in 11(c) and 11(d) are the relative areas of different grain boundary planes for the misorientation of $90^{\circ}$ about [10-10] and $30^{\circ}$ about [0001], respectively. The plots are stereographic projections. The maxima show that the $90^{\circ}[10-10]$ misorientation boundaries are concentrated on [10-10] planes, indicating that they are pure twist boundaries and the $30^{\circ}[0001]$ boundaries are concentrated on [0001], indicating that they are also pure twist boundaries. The local maxima at the prismatic orientations correspond to asymmetric tilt boundaries. One final representation is to consider the distribution of grain boundary planes alone, with no reference to the misorientation. This is the two parameter interface plane distribution. This type of distribution for WC/Co interfaces was already illustrated in Fig. 6(b).

Describing the GBCD with a reduced number of parameters is often useful because the complete five-parameter space of grain boundary types is large; if the space is discretized in $10^{\circ}$ intervals, then there are roughly $6 \times 10^{3}$ different grain boundaries for a material with cubic symmetry. (Rohrer et al., 2004) The number of distinct boundaries increases rapidly for finer discretizations and crystals with reduced symmetry. So, until recently, the number of distinct grain boundary types 
was large compared to the number of observations it was possible to make. However, this is no longer the case. The development of high-speed electron backscatter diffraction mapping of orientations has made it possible to characterize and classify $10^{4}$ to $10^{5}$ boundaries. (Adams et al., 1993, Schwartz et al., 2009)

\subsection{Interface topology}

While there have been many efforts during the past several years to characterize the GBCD, fewer studies have attempted to characterize the connectivity of grain boundaries in the microstructure. The statistics of triple junctions, twin related clusters, and percolation characteristics have all been explored as metrics for connenctivity (Frary and Schuh, 2003, Frary and Schuh, 2004, Frary and Schuh, 2005, Kumar et al., 2000, Minich et al., 2002, Reed et al., 2008, Schuh et al., 2003, Xia et al., 2006, Xia et al., 2008), and Schuh et al. (Schuh et al., 2003) found that boundary populations is not a reliable indicator of network topology. While there is also a long history of applying the mathematical tools of topology to microstructures, there are still relatively few examples. (Gameiro et al., 2005, Steele, 1972, Mecke, 1996, Mecke and Sofonea, 1997, Mendoza et al., 2004, Mendoza et al., 2006, Wanner et al., 2010)

In two dimensions, there are two topological metrics that measure the number of independent pieces of the network (referred to as $\mathrm{B}_{0}$ ) and the number of closed loops (referred to as $\mathrm{B}_{1}$ ). (Wanner et al., 2010) In the context of plane sections of grain boundary networks, $\mathrm{B}_{0}$ measures groups of grain boundaries not connected to the rest of the network and $\mathrm{B}_{1}$ measures continuous, closed paths of grain 
boundaries or the number of grains. It has recently been proposed that the ratio of $\mathrm{B}_{0}$ to $\mathrm{B}_{1}$, or the inverse connectivity, is a suitable metric for network connectivity. (Rohrer and Miller, 2010) The ratio varies with the subset of boundaries considered and its variation as a function of the disorientation angle threshold is a characteristic of the type of microstructure, providing information beyond that available from relative area measurements.

As an example, Fig. 12 compares two Ni microstructures (reference and grain boundary engineered) after separating the $\Sigma 3^{\mathrm{n}}$ and non- $\Sigma 3^{\mathrm{n}}$ boundaries. (Rohrer and Miller, 2010) The $\Sigma 3^{\mathrm{n}}$ boundaries are $\Sigma 3, \Sigma 9$, and $\Sigma 27$, in coincident site lattice notation. The network of $\Sigma 3^{n}$ boundaries in the reference sample is clearly disconnected and this is reflected in an inverse connectivity that is greater than 1. On the other hand, the network of non- $\Sigma 3^{n}$ boundaries (Fig. 12b) is relatively connected and this is reflected in an inverse connectivity that is less than one and smaller than the value for the $\Sigma 3^{n}$ network. The situation is reversed for the grain boundary engineered sample. In this case, the inverse connectivity is less than 1 for the $\Sigma 3^{\mathrm{n}}$ boundaries. The inverse connectivity of the network of non- $\Sigma 3^{\mathrm{n}}$ boundaries is 16 times larger, indicating that it is relatively disconnected compared to the network of $\Sigma 3^{\mathrm{n}}$ boundaries. This suggests that the main impact of grain boundary engineering in this case is to increase the connectivity of twin related boundaries while simultaneously decreasing the connectivity of random boundaries.

$<$ Figure 12 near here $>$ 


\subsection{Curvature Distributions}

The distribution of interface curvatures is not commonly evaluated, but is important for understanding microstructure evolution and verifying theories for capillarity driven processes such as coarsening in multiphased mixtures and grain growth in single phase polycrystals. Most theories have been compared to experiments where only an average curvature was determined. (Rohrer, 2005) Using three-dimensional data, it is now possible to measure the spatial distribution of curvatures which are important for local morphological evolution. (Alkemper and Voorhees, 2001) For example, a recent study of the curvatures of grain faces in three dimensions demonstrated that mean field approaches to grain growth are flawed because the curvature and growth rate of a grain is strongly affected by the topology of it nearest neighbors. (Rowenhorst et al., 2010)

\section{Recent Advances in Microstructural Characterization Techniques}

\subsection{Three-dimensional atom probe}

Three-dimensional atom probe (3DAP) is an increasingly powerful technique for the analysis of composition and structure on the near-atomic scale. (Kelly and Miller, 2007, Miller and Forbes, 2009, Seidman, 2007) This technique is commonly referred to as "atomic probe tomography". However, this is somewhat misleading; while the method is used to visualize three-dimensional volumes, the underling principle is closer to serial sectioning than tomography. 3DAP instruments combine a field ion microscope with a mass spectrometer; as atoms are ionized and ejected from the sample, their trajectories are used to determine their original positions in 
the sample and their atomic masses are determined by mass spectroscopy. In the past, there were a number of aspects of atom probe technology that limited its applicability. First, the preparation of needle shaped specimens by etching was laborious and labor intensive and made it difficult to locate specific features of interest. Second, the analysis was very slow. Finally, it was essentially impossible to examine ceramics and other insulators by this technique. However, progress in several different areas have eliminated or at least minimized these problems.

The dual beam focused ion beam (FIB) scanning electron microscope (SEM) has made it feasible to create samples of specific areas for analysis in the 3DAP. For example, single grain boundaries can be selected for analysis and the FIB can be used to mill a needle shape specimen that contains the interface or region of interest. The introduction of the local electrode and a position sensitive area detector greatly increased both the efficiency and the rate of ion collection. Finally, using a pulsed laser to assist with ionization made it possible to analyze insulators. As the wavelengths of the lasers are reduced and the pulse rates are increase (approaching a megahertz) the rate of data collection has also greatly increased. With these latest advances, it is possible to image $\sim 10^{8}$ atoms in a period of hours, approximately $50 \%$ of all of the ionized atoms are analyzed, the composition sensitivity in the parts per million range, and spatial resolution is on the order of a nanometer.

As an example of these recent advancements to the study of ceramics and hard materials, laser assisted 3DAP has recent been used to study the composition of a grain boundary in carbon-doped alumina. (Marquis et al., 2010) A FIB was used to 
cut a needle shaped sample containing a grain boundary. As shown in Fig. 13, there is an $\sim 10 \mathrm{~nm}$ wide region near the grain boundary that is enriched in carbon. This work is a significant advance because it shows that oxides and other insulting ceramics can now be characterized by 3DAP. Therefore, future applications to ceramics and hard materials is promising.

$<$ Figure 13 near here $>$

\subsection{Electron tomography}

Three-dimensional TEM imaging by electron tomography has recently become more common in materials research. (Barnard et al., 2006, Batenburg et al., 2009, Mobus and Inkson, 2001, Weyland et al., 2006) As with other tomographies, it involves recording a series of images from different perspectives over a large angular range. In principle, images should be recorded at all possible orientations and in small increments (on the order of $1^{\circ}$ ); in practice, the geometry of the sample holder and the microscope usually limits the range of rotation. While this is nonideal and can lead to some artifacts, it does not prevent application of the method. After the images are recorded, the three-dimensional volume is reconstructed and visualized using computer algorithms developed for this purpose. For example, the image in Fig. 14 shows silica particles within a polymer/ceramics composite. (Ikeda et al., 2004) While this technique has not been widely applied to ceramics and hard materials, it will clearly be useful for understanding the structure of composites.

$<$ Figure 14 near here $>$ 
The most common contrast mechanism for electron tomography is high-angle annular dark-field (HAADF) imaging in the scanning TEM (STEM). (Robertson et al., 2011) Because this contrast mechanism is sensitive to variations in the atomic number, it creates clear phase contrast. However, electron tomography can also be carried out with bright field or dark field images, although competing contrast mechanisms can interfere with the three-dimensional reconstruction. It is also possible to create three-dimensional composition maps by coupling the images to electron energy loss or energy-dispersive spectroscopy. In addition to structural information in multiphased materials, electron tomography has also been used to study dislocation dynamics and magnetic domain structure. (Robertson et al., 2011) The resolution of this technique is on the order of $1 \mathrm{~nm}$.

\subsection{X-ray tomography}

The basic principles of tomography can also be applied to a set of transmission X-ray radiographs to reconstruct a three-dimensional volume. (Chen et al., 2010, Chino and Dunand, 2008) Contrast in the radiographs arises from differences in absorption coefficients, so it is generally applied to multiphased materials and composites. Compared to electron tomography, the imaging conditions are much more straightforward and there is no limit on the angular range. While the resolution is lower than electron tomography, it is also possible to image much larger volumes. The samples must be partially X-ray transparent so it is the absorption coefficient of the sample, along with the density, which controls the 
maximum sample size. There are commercial desktop instruments that are capable of creating three-dimensional images with resolutions in the 10s of microns range and volumes on the order of $10^{2} \mathrm{~mm}^{3}$. Using the greater penetrating power of the higher energy X-rays available at a synchrotron, larger samples can be imaged at resolutions below 10 microns. Furthermore, recent advances in the ability to focus X-rays has made it possible to increase the resolution of synchrotron tomography to approximately $20 \mathrm{~nm}$. (Robertson et al., 2011)

An example of X-ray tomography is shown in Fig. 15. The sample is a SiC fiber$\mathrm{SiC}$ matrix composite formed by the chemical vapor infiltration of a woven fiber preform. (Morales-Rodriguez et al., 2009) This process leaves porosity in the material and this creates density contrast that can be imaged. This example was constructed from 1200 separate projections imaged with an $85 \mathrm{kV}$ beam and a pixel size of 4.2 microns. The data can be analyzed to determine the volume fraction of pores, the connectivity of the pore space, and the tortuosity of the pores. Another example in which a ceramic/metal composite has been characterized is a study of damage accumulation around $\mathrm{SiC}$ particles in $\mathrm{SiC} /$ aluminum composites. (Silva et al., 2010, Williams et al., 2010) Mo-TiC composites have also been imaged by this technique at the European Synchrotron Radiation Facility ID19 beam line and has a pixel size of $280 \mathrm{~nm}$. (Cedat et al., 2009) Data sets of this type are valuable as input to simulations of materials properties. As the resolution increases, X-ray tomography is likely to become a widespread technique for the study of composites containing hard materials. 
$<$ Figure 15 near here>

\subsection{DB-FIB/EBSD}

Automated electron backscatter diffraction (EBSD), which makes it possible to accumulate a map of crystal orientations on a surface, has had a transformative effect on the study of grain boundaries in polycrystals (see Fig. 16). (Adams et al., 1993) It is currently possible measure several hundred orientations per second, enabling the determination of the shapes and orientations of thousands of grains in a reasonable amount of time. In orientation maps such as that shown in Fig. 16(c), four of the five grain boundary parameters can be specified: the lattice misorientation and the in plane component of the grain boundary plane orientation. The most direct way to make an absolute determination of the final component of the boundary plane orientation is remove a small amount of material (small compared to the grain size) and repeat the measurement; a process referred to as serial sectioning. Manual serial sectioning coupled with EBSD to determine grain boundary plane crystallography is technically demanding and time consuming. As a result, there are relatively few examples in the literature. (Randle and Davies, 2002, Rowenhorst et al., 2006, Rowenhorst et al., 2010, Rowenhorst and Voorhees, 2005, Saylor et al., 2004a, Saylor et al., 2002, Saylor et al., 2003b)

$<$ Figure 16 near here $>$

The experimental barriers imposed by serial sectioning have been significantly reduced by dual beam microscopes that contain both an electron beam 
for EBSD mapping, and a FIB for material removal. (Dillon and Rohrer, 2009b, Groeber et al., 2006, Khorashadizadeh et al., 2011, Konrad et al., 2006, Li et al., 2009, Rohrer et al., 2010, Uchic et al., 2006) The sequence of orientation mapping and removing thin layers can be fully automated so that data can be acquired without user intervention. This has enabled the collection of large three-dimensional images of materials from which statistical information can be derived. Examples of threedimensional images of ceramics are illustrated in Fig. 17. The three-dimensional images can also be used as the input to simulations of materials response. (Lewis et al., 2008, Rollett et al., 2010, Zaafarani et al., 2006) It should also be noted that the FIB can be used for serial sectioning and imaging experiments without collecting orientation data. This type of three-dimensional imaging is somewhat simpler and considerably more wide-spread. (MacSleyne et al., 2009, Singh et al., 2010)

$<$ Figure 17 near here>

These methods are easily applied to ceramics. For example, EBSD maps of $\mathrm{Y}_{2} \mathrm{O}_{3}$ were obtained on parallel layers separated by $200 \mathrm{~nm}$ (see Fig. 17(a)). (Dillon and Rohrer, 2009b) The choices of the in plane and between plane spacing of orientation points are important for the success of the measurement. It has been found that the spacing between planes should be no larger than $1 / 10^{\text {th }}$ of the mean grain size and the spacing of orientation points in the plane should be at least one half of the spacing between planes. The processing of the data is also very important for the accurate calculation of the GBCD. In each EBSD map, average orientations were determined for each grain larger than 10 pixels and the grain 
boundaries were then approximated by a set of line segments. It was also found that sub-pixel alignment of the parallel layers was essential to achieve accurate results. (Dillon and Rohrer, 2009b, Li et al., 2009, Rohrer et al., 2010) If it is assumed that in an equiaxed microstrucuture the triple lines should be randomly oriented, then their average orientation should be parallel to the milling direction. However, when the orientations of the triple lines are examined in the sample reference frame, they are typically biased. To correct for the effect, a rigid offset is applied to the positions in each layer so that the mean of the triple line direction is normal to the sample surface. In most cases, these adjustments are less than the pixel spacing.

The distribution of grain boundary planes in the yttria polycrystal for misorientations of $30^{\circ}$ and $60^{\circ}$ about [111] are shown in 18(a) and 18(b). (Dillon and Rohrer, 2009b) In each distribution, there is a peak at the twist position, where the grain boundary is bounded on both sides by a (111) plane. The Grain boundary energy distributions for the same misorientations are shown in Figs. 18(c) and (d). The energy shows an approximate inverse relationship to the distribution; the minimum energy occurs at the (111) twist position where the grain boundary plane distribution reaches a maximum. Based on these results, there is an inverse relationship between the grain population and the grain boundary energy. In fact, this inverse correlation persists throughout the domain of grain boundary types of all of the materials that have been examined. (Dillon and Rohrer, 2009b, Li et al., 2009, Rohrer, 2011a, Rohrer et al., 2010, Saylor et al., 2003a) This inverse correlation is thought to arise from the preferential elimination of higher energy boundaries during grain growth. (Dillon and Rohrer, 2009a, Rohrer, 2011a) 
$<$ Figure 18 near here $>$

Three-dimensional microstructural data is particularly important when the connectivity of a microstructure is important, as is the case for a solid oxide fuel cell. For example, in the active cathode region there is a three-phase mixture of lanthanum-strontium manganate (LSM), yttria stablilized zirconia (YSZ), and pore space. The oxidation of oxygen is thought to only happen at the three phase line where gaseous oxygen, oxide ion conducting YSZ, and electron conducting LSM meet. A representative section of data from this region is illustrated in Fig. 19. (Dillon et al., 2011, Helmick et al., 2011) The study showed that the triple phase lines preferred [111] orientations and that this did not change in service. (Dillon et al., 2011)

$<$ Figure 19 near here $>$

\subsection{X-ray diffraction microscopy}

$\mathrm{X}$-ray tomography makes it possible to map the internal structure of a material nondestructively, but in its normal mode provides no crystallographic information. Three-dimensional orientation maps obtained by combining serial sections of planar EBSD orientation maps to reconstruct a three-dimensional volume (section 3.4) provides crystallographic information, but is destructive and does not permit time dependent studies polycrystalline structure. There are, however, a number of emerging high-energy X-ray techniques that allow orientation maps to be 
determined from transmitted X-ray beams. (Hefferan et al., 2009, Johnson et al., 2008, Larson et al., 2002, Poulsen et al., 2001) This makes it possible to create a three-dimensional orientation map, subject the sample to external stimulus (mechanical, chemical, thermal, or electrical) and map the structure again.

The most promising X-ray orientation mapping methods are based on transmitted X-rays and, because of this, high energy (50-100kV) synchrotron radiation is necessary to achieve penetrations depths of millimeters to centimeters. High energy diffraction microscopy uses multiple detectors to determine a diffracted beam's origin and, therefore, the physical location of each diffracting grain within a sample; it then combines information about the shapes of diffraction spots and the relative positions of diffraction spots to determine grain shape and orientation, respectively. (Poulsen et al., 2001) Diffraction contrast tomography (DCT) is closely related to HEDM, but is a tomographic technique.(Johnson et al., 2008) In X-ray tomography, the contrast is determined by density differences. However, if a monochromatic beam is used and the individual crystals are sufficiently large, then a portion of the transmitted beam is diffracted away, and this creates contrast in the radiograph that can be associated with the projected grain shape and location; the diffraction angles can be used to determine the grain orientation. This technique has been used to study the paths followed by cracks that advance by stress corrosion cracking. (King et al., 2008)

These techniques currently have resolutions in the range of 1-5 microns and have yet to be widely applied. One published application of DCT to a ceramic is a study of $\mathrm{SrTiO}_{3}$ and an example of the results is shown in Fig. 20. (Syha et al., 2012) 
This sample contains more than 800 grains and about half are internal and do not interact with the surface. The data can be used to determine both the grain and pore size distributions. In addition, the grain shapes and topologies can be determined. For example, the average number of faces per grain for the sample in Fig. 20 is 12.8. Furthermore, because the data includes crystallographic information all textural data and the five parameter GBCD can also be calculated.

$<$ Figure 20 near here $>$

\section{Summary and Conclusions}

Section 2 of this chapter summarized quantitative parameters that could be derived from microstructural characterization efforts. The recent implementation of three-dimensional microstructure characterization has led to both increasingly sophisticated and increasingly accurate descriptions of grain size distributions, grain shape descriptions, interface character distributions, and microstructural connectivity. This has mainly been enabled by the development of the advanced three-dimensional characterization techniques that were described in section 3. While not all of these have been applied widely to ceramics or other hard materials, they are applicable and will certainly have impact in the future.

There are three current trends in microstructural characterization that are likely to continue for some time. First, the numbers of features characterized will increase, structures will be characterized in three dimensions, and, in some cases, three-dimensional structures will be characterized as a function of time. Larger data sets will allow a representative volume of the material to be quantified, and 
three-dimensional and time dependent data will allow mechanisms to be explored.

The second trend is that structure data will be used as input for simulations of materials properties. Such studies will be necessary to validate simulations that can be used to study hypothetical structures. Finally, structural characterization will increasingly use multiple techniques to span length scales. It is anticipated that these trends will lead to increasingly powerful structure-property relationships for a wide range of ceramic and composite hard materials.

\section{Acknowledgement}

The work was supported by the MRSEC program of the National Science Foundation under Award Number DMR-0520425

\section{References}

Adams, B. L., Wright, S. I. \& Kunze, K. 1993. Orientation imaging - the emergence of a new microscopy. Metallurgical Transactions A-Physical Metallurgy and Materials Science, 24, 819-831.

Alkemper, J. \& Voorhees, P. W. 2001. Three-dimensional characterization of dendritic microstructures. Acta Materialia, 49, 897-902.

Bando, Y. 1995. Microstructure analysis of advanced ceramics by high-resolution analytical transmission electron-microscopy. Journal of Electron Microscopy, 44, 115-123.

Barnard, J. S., Sharp, J., Tong, J. R. \& Midgley, P. A. 2006. High-resolution threedimensional imaging of dislocations. Science, 313, 319-319.

Batenburg, K. J., Bals, S., Sijbers, J., Kubel, C., Midgley, P. A., Hernandez, J. C., Kaiser, U., Encina, E. R., Coronado, E. A. \& Van Tendeloo, G. 2009. 3D imaging of nanomaterials by discrete tomography. Ultramicroscopy, 109, 730-740.

Cedat, D., Libert, M., Le Flem, M., Fandeur, O., Rey, C., Clavel, M. \& Schmitt, J. H. 2009. Experimental characterization and mechanical behaviour modelling of molybdenum-titanium carbide composite for high temperature applications. International Journal of Refractory Metals \& Hard Materials, 27, 267-273.

Chen, Y. C. K., Chu, Y. S., Yi, J., Mcnulty, I., Shen, Q., Voorhees, P. W. \& Dunand, D. C. 2010. Morphological and topological analysis of coarsened nanoporous gold by X-ray nanotomography. Applied Physics Letters, 96. 
Chien, H., Gao, M. C., Miller, H. M., Rohrer, G. S., Ban, Z. G., Prichard, P. \& Liu, Y. X. 2009. Microtexture and hardness of cvd deposited alpha- $\mathrm{Al}_{2} \mathrm{O}_{3}$ and $\mathrm{TiC}_{\mathrm{x}} \mathrm{N}_{1-\mathrm{x}}$ coatings. International Journal of Refractory Metals \& Hard Materials, 27, 458464.

Chino, Y. \& Dunand, D. C. 2008. Directionally freeze-cast titanium foam with aligned, elongated pores. Acta Materialia, 56, 105-113.

Dillon, S. \& Rohrer, G. 2009a. Mechanism for the development of anisotropic grain boundary character distributions during normal grain growth. Acta Materialia, 57, 1-7.

Dillon, S. J., Helmick, L., Miller, H. M., Wilson, L., Gemman, R., Petrova, R. V., Barmak, K., Rohrer, G. S. \& Salvador, P. A. 2011. The orientation distributions of lines, surfaces, and interfaces around three-phase boundaries in solid oxide fuel cell cathodes. Journal of the American Ceramic Society, 94, 4045-4051.

Dillon, S. J. \& Rohrer, G. S. 2009b. Characterization of the grain-boundary character and energy distributions of yttria using automated serial sectioning and EBSD in the FIB. Journal of the American Ceramic Society, 92, 1580-1585.

Frary, M. \& Schuh, C. A. 2003. Nonrandom percolation behavior of grain boundary networks in high- $\mathrm{T}_{\mathrm{c}}$ superconductors. Applied Physics Letters, 83, 3755-3757.

Frary, M. \& Schuh, C. A. 2004. Percolation and statistical properties of low- and highangle interface networks in polycrystalline ensembles. Physical Review B, 69, 12.

Frary, M. \& Schuh, C. A. 2005. Connectivity and percolation behaviour of grain boundary networks in three dimensions. Philosophical Magazine, 85, 11231143.

French, D. N. 1969. Microstructure and microproperties of wc-co alloys. International Journal of Powder Metallurgy, 5, 47-60.

Gameiro, M., Mischaikow, K. \& Wanner, T. 2005. Evolution of pattern complexity in the Cahn-Hilliard theory of phase separation. Acta Materialia, 53, 693-704.

German, R. M. 1985. The contiguity of liquid-phase sintered microstructures. Metallurgical Transactions A-Physical Metallurgy and Materials Science, 16, 1247-1252.

Groeber, M. A., Haley, B. K., Uchic, M. D., Dimiduk, D. M. \& Ghosh, S. 2006. 3D reconstruction and characterization of polycrystalline microstructures using a fib-sem system. Materials Characterization, 57, 259-273.

Gurland, J. 1988. New scientific approaches to development of tool materials. International Materials Reviews, 33, 151-166.

Hefferan, C. M., Li, S. F., Lind, J., Lienert, U., Rollett, A. D., Wynblatt, P. \& Suter, R. M. 2009. Statistics of high purity nickel microstructure from high energy x-ray diffraction microscopy. CMC-Computers Materials \& Continua, 14, 209-219.

Helmick, L., Dillon, S. J., Gerdes, K., Gemmen, R., Rohrer, G. S., Seetharaman, S. \& Salvador, P. A. 2011. Crystallographic characteristics of grain boundaries in dense yttria-stabilized zirconia. International Journal of Applied Ceramic Technology, 8, 1218-1228.

Hollox, G. E. 1968. Microstructure and mechanical behavior of carbides. Materials Science and Engineering, 3, 121-137. 
Hull, F. C. 1988. Plane section and spatial characteristics of equiaxed beta-brass grains. Materials Science and Technology, 4, 778-785.

Ikeda, Y., Katoh, A., Shimanuki, J. \& Kohjiya, S. 2004. Nano-structural observation of in situ silica in natural rubber matrix by three dimensional transmission electron microscopy. Macromolecular Rapid Communications, 25, 1186-1190.

Ikuhara, Y. 2001. Grain boundary and interface structures in ceramics. Journal of the Ceramic Society of Japan, 109, S110-S120.

Jha, S. K., Larsen, J. M. \& Rosenberger, A. H. 2009. Towards a physics-based description of fatigue variability behavior in probabilistic life-prediction. Engineering Fracture Mechanics, 76, 681-694.

Johnson, G., King, A., Honnicke, M. G., Marrow, J. \& Ludwig, W. 2008. X-ray diffraction contrast tomography: A novel technique for three-dimensional grain mapping of polycrystals. II. The combined case. Journal of Applied Crystallography, 41, 310-318.

Kelly, T. F. \& Miller, M. K. 2007. Invited review article: Atom probe tomography. Review of Scientific Instruments, 78, 031101, 1-20.

Khorashadizadeh, A., Raabe, D., Zaefferer, S., Rohrer, G. S., Rollett, A. D. \& Winning, M. 2011. Five-parameter grain boundary analysis by 3D EBSD of an ultra fine grained CuZr alloy processed by equal channel angular pressing. Advanced Engineering Materials, 13, 237-244.

Kim, C. S., Massa, T. R. \& Rohrer, G. S. 2008. Interface character distributions in WCCo composites. Journal of the American Ceramic Society, 91, 996-1001.

Kim, C. S. \& Rohrer, G. S. 2004. Geometric and crystallographic characterization of wc surfaces and grain boundaries in WC-Co composites. Interface Science, 12, 19-27.

King, A., Johnson, G., Engelberg, D., Ludwig, W. \& Marrow, J. 2008. Observations of intergranular stress corrosion cracking in a grain-mapped polycrystal. Science, 321, 382-385.

Kleebe, H. J., Braue, W., Schmidt, H., Pezzotti, G. \& Ziegler, G. 1996. Transmission electron microscopy of microstructures in ceramic materials. Journal of the European Ceramic Society, 16, 339-351.

Konrad, J., Zaefferer, S. \& Raabe, D. 2006. Investigation of orientation gradients around a hard laves particle in a warm-rolled $\mathrm{Fe}_{3} \mathrm{Al}$-based alloy using a 3D EBSD-FIB technique. Acta Materialia, 54, 1369-1380.

Kral, M. V. \& Spanos, G. 1999. Three-dimensional analysis of proeutectoid cementite precipitates. Acta Materialia, 47, 711-724.

Kumar, M., King, W. E. \& Schwartz, A. J. 2000. Modifications to the microstructural topology in f.C.C. Materials through thermomechanical processing. Acta Materialia, 48, 2081-2091.

Larson, B. C., Yang, W., Ice, G. E., Budai, J. D. \& Tischler, J. Z. 2002. Three-dimensional $\mathrm{X}$-ray structural microscopy with submicrometre resolution. Nature, 415, 887-890.

Lewis, A. C., Jordan, K. A. \& Geltmacher, A. B. 2008. Determination of critical microstructural features in an austenitic stainless steel using image-based finite element modeling. Metallurgical and Materials Transactions A-Physical Metallurgy and Materials Science, 39A, 1109-1117. 
Li, J., Dillon, S. J. \& Rohrer, G. S. 2009. Relative grain boundary area and energy distributions in nickel. Acta Materialia, 57, 4304-4311.

Luyckx, S. \& Love, A. 2006. The dependence of the contiguity of WC on Co content and its independence from wc grain size in WC-Co alloys. International Journal of Refractory Metals \& Hard Materials, 24, 75-79.

Macsleyne, J., Uchic, M. D., Simmons, J. P. \& De Graef, M. 2009. Three-dimensional analysis of secondary gamma-prime precipitates in rene-88 DT and UMF-20 superalloys. Acta Materialia, 57, 6251-6267.

Marquis, E. A., Yahya, N. A., Larson, D. J., Miller, M. K. \& Todd, R. I. 2010. Probing the improbable: Imaging C atoms in alumina. Materials Today, 13, 34-36.

Mecke, K. R. 1996. Morphological characterization of patterns in reaction-diffusion systems. Physical Review E, 53, 4794-4800.

Mecke, K. R. \& Sofonea, V. 1997. Morphology of spinodal decomposition. Physical Review E, 56, R3761-R3764.

Mendoza, R., Savin, I., Thornton, K. \& Voorhees, P. W. 2004. Topological complexity and the dynamics of coarsening. Nature Materials, 3, 385-388.

Mendoza, R., Thornton, K., Savin, I. \& Voorhees, P. W. 2006. The evolution of interfacial topology during coarsening. Acta Materialia, 54, 743-750.

Miller, M. K. \& Forbes, R. G. 2009. Atom probe tomography. Materials Characterization, 60, 461-469.

Minich, R. W., Schuh, C. A. \& Kumar, M. 2002. Role of topological constraints on the statistical properties of grain boundary networks. Physical Review B, 66, 4.

Mobus, G. \& Inkson, B. J. 2001. Three-dimensional reconstruction of buried nanoparticles by element-sensitive tomography based on inelastically scattered electrons. Applied Physics Letters, 79, 1369-1371.

Morales-Rodriguez, A., Reynaud, P., Fantozzi, G., Adrien, J. \& Maire, E. 2009. Porosity analysis of long-fiber-reinforced ceramic matrix composites using x-ray tomography. Scripta Materialia, 60, 388-390.

National Insititiutes of Health, Imagej. http://rsb.info.nih.gov/ij/

Pollock, T. M., Allison, J. E., Backman, D. G., Boyce, M. C., Gersh, M., Holm, E. A., Lesar, R., Long, M., Powell, A. C., Schirra, J. J., Demania Whitis, D. \& Woodward, C. 2008. Integrated computational materials engineering: a transformational discipline for improved competitiveness and national security, Washington, DC, National Academy of Sciences.

Poulsen, H. F., Nielsen, S. F., Lauridsen, E. M., Schmidt, S., Suter, R. M., Lienert, U., Margulies, L., Lorentzen, T. \& Jensen, D. J. 2001. Three-dimensional maps of grain boundaries and the stress state of individual grains in polycrystals and powders. Journal of Applied Crystallography, 34, 751-756.

Randle, V. \& Davies, H. 2002. A comparison between three-dimensional and twodimensional grain boundary plane analysis. Ultramicroscopy, 90, 153-162.

Reed, B. W., Kumar, M., Minich, R. W. \& Rudd, R. E. 2008. Fracture roughness scaling and its correlation with grain boundary network structure. Acta Materialia, 56, 3278-3289.

Robertson, I. M., Schuh, C. A., Vetrano, J. S., Browning, N. D., Field, D. P., Jensen, D. J., Miller, M. K., Baker, I., Dunand, D. C., Dunin-Borkowski, R., Kabius, B., Kelly, T., Lozano-Perez, S., Misra, A., Rohrer, G. S., Rollett, A. D., Taheri, M. L., 
Thompson, G. B., Uchic, M., Wang, X.-L. \& Was, G. 2011. Towards an integrated materials characterization toolbox. Journal of Materials Research, 26, 1341-1383.

Rohrer, G. S. 2005. Influence of interface anisotropy on grain growth and coarsening. Annual Review of Materials Research, 35, 99-126.

Rohrer, G. S. 2011a. Grain boundary energy anisotropy: A review. Journal of Materials Science, 46, 5881-5895.

Rohrer, G. S. 2011b. Measuring and interpreting the structure of grain-boundary networks. Journal of the American Ceramic Society, 94, 633-646.

Rohrer, G. S., Li, J., Lee, S., Rollett, A. D., Groeber, M. \& Uchic, M. D. 2010. Deriving grain boundary character distributions and relative grain boundary energies from three-dimensional ebsd data. Materials Science and Technology, 26, 661-669.

Rohrer, G. S. \& Miller, H. M. 2010. Topological characteristics of plane sections of polycrystals. Acta Materialia, 58, 3805-3814.

Rohrer, G. S., Saylor, D. M., El Dasher, B., Adams, B. L., Rollett, A. D. \& Wynblatt, P. 2004. The distribution of internal interfaces in polycrystals. Zeitschrift Fur Metallkunde, 95, 197-214.

Rollett, A. D., Lebensohn, R. A., Groeber, M., Choi, Y., Li, J. \& Rohrer, G. S. 2010. Stress hot spots in viscoplastic deformation of polycrystals. Modelling and Simulation in Materials Science and Engineering, 18.

Rowenhorst, D. J., Gupta, A., Feng, C. R. \& Spanos, G. 2006. 3D crystallographic and morphological analysis of coarse martensite: Combining EBSD and serial sectioning. Scripta Materialia, 55, 11-16.

Rowenhorst, D. J., Lewis, A. C. \& Spanos, G. 2010. Three-dimensional analysis of grain topology and interface curvature in a beta-titanium alloy. Acta Materialia, 58, 5511-5519.

Rowenhorst, D. J. \& Voorhees, P. W. 2005. Measurements of the grain boundary energy and anisotropy in tin. Metallurgical and Materials Transactions APhysical Metallurgy and Materials Science, 36A, 2127-2135.

Russ, J. C. 1986. Practical stereology, Springer.

Sano, T., Kim, C. S. \& Rohrer, G. S. 2005. Shape evolution of $\mathrm{SrTiO}_{3}$ crystals during coarsening in titania-rich liquid. Journal of the American Ceramic Society, 88, 993-996.

Saylor, D., Morawiec, A. \& Rohrer, G. 2003a. The relative free energies of grain boundaries in magnesia as a function of five macroscopic parameters. Acta Materialia, 51, 3675-3686.

Saylor, D. M., El Dasher, B., Sano, T. \& Rohrer, G. S. 2004a. Distribution of grain boundaries in $\mathrm{SrTiO}_{3}$ as a function of five macroscopic parameters. Journal of the American Ceramic Society, 87, 670-676.

Saylor, D. M., El-Dasher, B. S., Adams, B. L. \& Rohrer, G. S. 2004b. Measuring the fiveparameter grain-boundary distribution from observations of planar sections. Metallurgical and Materials Transactions A-Physical Metallurgy and Materials Science, 35A, 1981-1989. 
Saylor, D. M., Morawiec, A. \& Rohrer, G. S. 2002. Distribution and energies of grain boundaries in magnesia as a function of five degrees of freedom. Journal of the American Ceramic Society, 85, 3081-3083.

Saylor, D. M., Morawiec, A. \& Rohrer, G. S. 2003b. Distribution of grain boundaries in magnesia as a function of five macroscopic parameters. Acta Materialia, 51, 3663-3674.

Saylor, D. M. \& Rohrer, G. S. 2002. Determining crystal habits from observations of planar sections. Journal of the American Ceramic Society, 85, 2799-2804.

Schuh, C. A., Kumar, M. \& King, W. E. 2003. Analysis of grain boundary networks and their evolution during grain boundary engineering. Acta Materialia, 51, 687700.

Schwartz, A. J., Kumar, M., Adams, B. L. \& Field, D. P. 2009. Electron backscatter diffraction in materials science, New York, Springer.

Seidman, D. N. 2007. Three-dimensional atom-probe tomography: Advances and applications. Annual Review of Materials Research, 37, 127-158.

Sherman, D. \& Brandon, D. 1999. Mechanical properties of hard materials and their relation to microstructure. Advanced Engineering Materials, 1, 161-181.

Silva, F. D., Williams, J. J., Muller, B. R., Hentschel, M. P., Portella, P. D. \& Chawla, N. 2010. Three-dimensional microstructure visualization of porosity and Ferich inclusions in $\mathrm{SiC}$ particle-reinforced al alloy matrix composites by X-ray synchrotron tomography. Metallurgical and Materials Transactions A-Physical Metallurgy and Materials Science, 41A, 2121-2128.

Singh, D. R. P., Chawla, N. \& Shen, Y. L. 2010. Focused ion beam (FIB) tomography of nanoindentation damage in nanoscale metal/ceramic multilayers. Materials Characterization, 61, 481-488.

Smith, M., Garcia, R. E. \& Horn, Q. C. 2009. The effect of microstructure on the galvanostatic discharge of graphite anode electrodes in $\mathrm{LiCoO}_{2}$-based rocking-chair rechargeable batteries. Journal of the Electrochemical Society, 156, A896-A904.

Spowart, J. E., Mullens, H. M. \& Puchala, B. T. 2003. Collecting and analyzing microstructures in three dimensions: A fully automated approach. JOMJournal of the Minerals Metals \& Materials Society, 55, 35-37.

Steele, J.H. 1972. Application of topological concepts in stereology. In: Pellissier, G. E. Purdy, S.M. (ed.) Stereology and quantitative metallography. Philadelphia, PA: American Society for Testing and Materials.

Syha, M., Rheinheimer, W., Baurer, M., Lauridsen, E. M., Ludwig, W., Weygand, D. \& Gumbsch, P. 2012. Three-dimensional grain structure of sintered bulk strontium titanate from x-ray diffraction contrast tomography. Scripta Materialia, 66, 1-4.

Thomas, G. 1996. Electron microscopy and microanalysis of ceramics. Journal of the European Ceramic Society, 16, 323-338.

Tucker, J. C., Chan, L. H., Rohrer, G. S., Groeber, M. A. \& Rollett, A. D. 2012. Tail departure of log-normal grain size distributions in synthetic threedimensional microstructures. Metallurgical and Materials Transactions A. Available on line, DOI: 10.1007/s11661-011-0851-4 
Tucker, M. O. \& Mclachlan, N. 1993. Fracture and microstructure of graphites. Journal of Physics D-Applied Physics, 26, 893-907.

Uchic, M. D., Groeber, M. A., Dimiduk, D. M. \& Simmons, J. P. 2006. 3D microstructural characterization of nickel superalloys via serial-sectioning using a dual beam FIB-SEM. Scripta Materialia, 55, 23-28.

Varela, M., Lupini, A. R., Van Benthem, K., Borisevich, A. Y., Chisholm, M. F., Shibata, N., Abe, E. \& Pennycook, S. J. 2005. Materials characterization in the aberration-corrected scanning transmission electron microscope. Annual Review of Materials Research, 35, 539-569.

Wanner, T., Fuller, E. R. \& Saylor, D. M. 2010. Homology metrics for microstructure response fields in polycrystals. Acta Materialia, 58, 102-110.

Weyland, M., Yates, T. J. V., Dunin-Borkowski, R. E., Laffont, L. \& Midgley, P. A. 2006. Nanoscale analysis of three-dimensional structures by electron tomography. Scripta Materialia, 55, 29-33.

White House Office of Science, Technology, and Policy. 2011. Materials genome initiative for global competitiveness. http://www.whitehouse.gov/sites/default/files/microsites/ostp/materials_ genome_initiative-final.pdf

Williams, J. J., Flom, Z., Amell, A. A., Chawla, N., Xiao, X. \& De Carlo, F. 2010. Damage evolution in $\mathrm{SiC}$ particle reinforced al alloy matrix composites by x-ray synchrotron tomography. Acta Materialia, 58, 6194-6205.

Williams, W. M. \& Smith, C. S. 1952. A study of grain shape in an aluminum alloy and other applications of stereoscopic microradiography. Journal of Metals, $\mathbf{4}$, 755-765.

Wilson, J. R., Kobsiriphat, W., Mendoza, R., Chen, H. Y., Hiller, J. M., Miller, D. J., Thornton, K., Voorhees, P. W., Adler, S. B. \& Barnett, S. A. 2006. Threedimensional reconstruction of a solid-oxide fuel-cell anode. Nature Materials, 5, 541-544.

Xia, S., Zhou, B. X., Chen, W. J. \& Wang, W. G. 2006. Effects of strain and annealing processes on the distribution of sigma 3 boundaries in a ni-based superalloy. Scripta Materialia, 54, 2019-2022.

Xia, S. A., Zhou, B. X. \& Chen, W. J. 2008. Effect of single-step strain and annealing on grain boundary character distribution and intergranular corrosion in alloy 690. Journal of Materials Science, 43, 2990-3000.

Zaafarani, N., Raabe, D., Singh, R. N., Roters, F. \& Zaefferer, S. 2006. Threedimensional investigation of the texture and microstructure below a nanoindent in a cu single crystal using 3D EBSD and crystal plasticity finite element simulations. Acta Materialia, 54, 1863-1876.

\section{Figure Captions}


Figure 1. Approximate limitations on the numbers of grains that can be mapped in three dimensions using selected techniques. The bounds of the domains of these techniques are expected to expand to the right with time. (Rohrer, 2011b)

Figure 2. Contact AFM image of a WC/Co sample with a carbide volume fraction of 0.82. The lightest contrast corresponds to Co and the carbide crystals are a variety of darker shades. (b) A skeletonized map determined from the AFM image by tracing the boundary positions. The average grain diameter is $1.53 \mu \mathrm{m}$ and the contiguity of the carbide phase is 0.50 . (Kim et al., 2008)

Figure 3. (a) The carbide grain size distribution of a WC/Co sample with a carbide volume fraction of 0.83 . (b) The relationship between the mean grain diameter and standard deviation of the distribution of grain sizes for a range specimens with different volume fractions and different grain sizes. (Kim et al., 2008)

Figure 4. Distribution of $\sim 5800$ grain sizes measured in three dimensions compared to a lognormal distribution (blue line). Red lines are drawn one standard deviation about the mean. The largest grains deviate from this distribution (purple circle). (Tucker et al., 2012)

Figure 5. (a) Electron backscatter diffraction orientation map for a $\mathrm{TiC}_{\mathrm{x}} \mathrm{N}_{1-\mathrm{x}}$ coating grown by chemical vapor deposition. (b) This is a transverse section of the coating, 
as indicated in this schematic. (c) Each color corresponds to a crystal orientation, defined by this key.(Chien et al., 2009)

Figure 6. (a) An atomic force microscope image of a WC/Co composite after etching. The dark polygonal features are WC grains. (b) Interface plane distribution for the WC/Co interfaces in a WC/Co composite, showing that particles are dominantly bound by basal and prismatic surfaces. (c) Average base-to-height aspect ratios $(\mathrm{b} / \mathrm{h})$ for the carbide grains in the seven samples, plotted as a function of carbide volume fraction. (Kim et al., 2008)

Figure 7. The base-to-height aspect ratio of WC crystals in a WC/Co sample with a carbide volume fraction of 0.85 , determined using different numbers of observations. The result converges after 500 line segments. (Kim et al., 2008)

Figure 8. Plot of a moment invariant, $\bar{\Omega}_{3}$, as a function of a shape parameter, $\mathrm{Q}$, for the $260 \gamma^{\prime}$ precipitates from a Rene' 88 -DT superalloy. The color scheme corresponds to the particle volume. The precipitates shown in the inset correspond to the black circles. The horizontal dashed line indicates the location of the class of rectangular prisms, with the cube located at the position indicated on the far right. From (MacSleyne et al., 2009). Copyright Elsevier, reproduced with permission.

Figure 9. The WC contiguity as a function of the carbide volume fraction for seven different WC/Co specimens. (Kim et al., 2008) 
Figure 10. (a) Electron backscatter diffraction orientation map of a transverse section of an $\alpha-\mathrm{Al}_{2} \mathrm{O}_{3}$ coating grown by chemical vapor deposition (color legend for the orientation is shown on the left). (b) Inverse pole figure for the growth direction of the sample. (c) Pole figure for the [0001] directions in the crystal. (Chien et al., 2009)

Figure 11. Three different representations of the distribution of relative areas of different types of grain boundaries. (a) Each boundary is classified by its minimum misorientation angle, without consideration of the misorientation axis. (b) Each position in this three-dimensional axis-angle space corresponds to a different misorientation. For any particular axis-angle combination, there is a distribution of grain boundary planes and the distributions for the two peaks in (b) are shown in (c) and (d). (Rohrer, 2011b)

Figure 12. Grain boundary maps for the Ni samples. (a) All of the $\Sigma 3^{\mathrm{n}}$ boundaries in the low $\Sigma 3 \mathrm{Ni}, \beta_{01}=3.1$, (b) all of the non $\Sigma 3^{\mathrm{n}}$ boundaries in the low $\Sigma 3 \mathrm{Ni}, \beta_{01}=$ 0.12 , (c) all of the $\Sigma 3^{\mathrm{n}}$ boundaries in high $\Sigma 3 \mathrm{Ni}, \beta_{01}=0.53$, (d) All of the non- $\Sigma 3^{\mathrm{n}}$ boundaries in high $\Sigma 3 \mathrm{Ni}, \beta_{01}=$ 8.6. In (a) and (c) the $\Sigma 3$ boundaries are colored red, the $\Sigma 9$ boundaries are blue and the $\Sigma 27$ boundaries are green. (Rohrer and Miller, 2010) 
Figure 13. 3DAP analysis of a grain boundary in carbon-doped alumina. (a) SEM image of the etched surface revealing grain boundaries. (b) FIB prepared 3DAP specimen containing a grain boundary near the apex of the specimen; (c) atomprobe reconstruction showing carbon segregation at the grain boundary; (d) Concentration profile across grain boundary along the red arrow shown in b). From (Marquis et al., 2010). Copyright Elsevier, reproduced with permission.

Figure 14. Volume rendered view of the reconstructed mass density distribution of silica inclusions in a rubber matrix. The frame is shown in reconstructed perspective geometry (length and width: $630 \mathrm{~nm}$, thickness: $181 \mathrm{~nm}$ ). The bar for each direction shows the distance of $100 \mathrm{~nm}$. From (Ikeda et al., 2004). Copyright John Wiley and Sons, reproduced with permission.

Figure 15. Three-dimensional volume rendering of the axial view of pores in twodimensional $\mathrm{SiC}_{\mathrm{f}}-\mathrm{SiC}$ composites. The dimensions of the reconstructed threedimensional volume is $\mathrm{x} \sim 2.5 \mathrm{~mm}, \mathrm{y} \sim 1.5 \mathrm{~mm}$ and $\mathrm{z} \sim 3.8 \mathrm{~mm}$ (total volume $\sim 14.3$ $\mathrm{mm}^{3}$ ). From (Morales-Rodriguez et al., 2009). Copyright Elsevier, reproduced with permission.

Figure 16. (a) Electron backscatter diffraction (EBSD) maps are recorded in SEM with the sample tilted at a large angle with respect to the beam. (b) A digital camera captures a diffraction pattern that is characteristic of the volume of material that interacted with the beam. (c) After recording local orientations on a grid of points, 
an orientation map can be plotted. The orientations of the $\mathrm{TiO}_{2}$ sample are specified by colors defined in the legend. (Rohrer, 2011b)

Figure 17. (a) A three-dimensional orientation map of yttria $\left(\mathrm{Y}_{2} \mathrm{O}_{3}\right)$ based on 43 parallel EBSD maps. (Dillon and Rohrer, 2009a) (b) A three-dimensional orientation map of $8 \%$ yttria $\left(\mathrm{Y}_{2} \mathrm{O}_{3}\right)$ stabilized Zirconia $\left(\mathrm{ZrO}_{2}\right)$. (Helmick et al., 2011)

Figure 18. (a-b) The grain-boundary plane distribution at the fixed misorientations of $30^{\circ} /[111]$ and $60^{\circ} /[111]$, plotted in stereographic projection along [001]. The corresponding energy distributions are shown in (c) and (d). (Dillon and Rohrer, 2009a)

Figure 19. Oblique projections of the reconstructed three-dimensional volume of the active cathode of a solid oxide fuel cell. In the orientation map in (a), different colors are used to indicate different grains. In the phase map (b), green indicates YSZ, gold indicates LSM, and red indicates pores. (Helmick et al., 2011)

Figure 20. Three-dimension view of a $\mathrm{SrTiO}_{3}$ polycrystal (a) (colored randomly) and cross-section (colored according to crystallographic orientation) (b) of the reconstructed structure compared to a SEM micrograph (c) of the same material. Collective ensemble of intergranular pores (d). (Syha et al., 2012) Copyright Elsevier, reproduced with permission. 


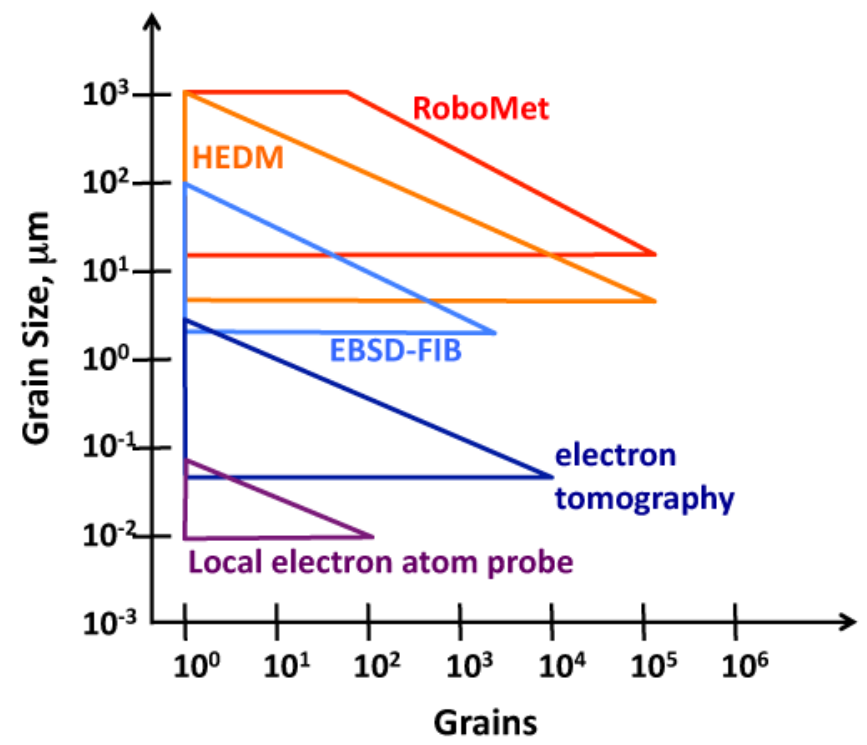

Figure 1. Approximate limitations on the numbers of grains that can be mapped in three dimensions using selected techniques. The bounds of the domains of these techniques are expected to expand to the right with time. (Rohrer, 2011b)
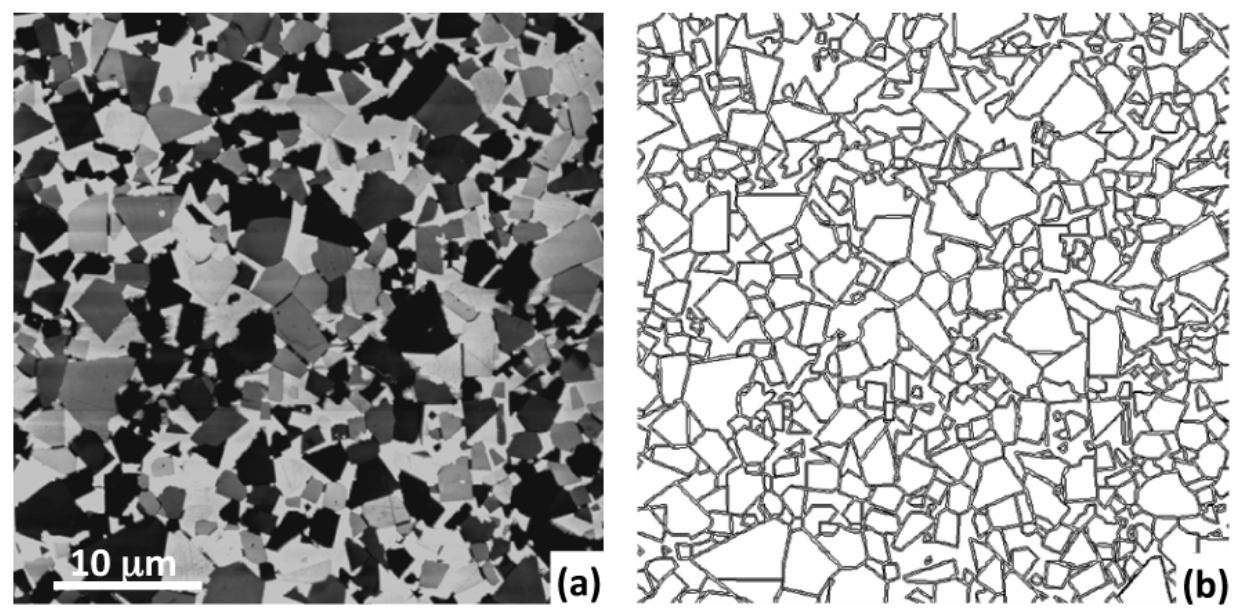

Figure 2. Contact AFM image of a WC/Co sample with a carbide volume fraction of 0.82. The lightest contrast corresponds to Co and the carbide crystals are a variety of darker shades. (b) A skeletonized map determined from the AFM image by tracing the boundary positions. The average grain diameter is $1.53 \mu \mathrm{m}$ and the contiguity of the carbide phase is 0.50 . (Kim et al., 2008) 


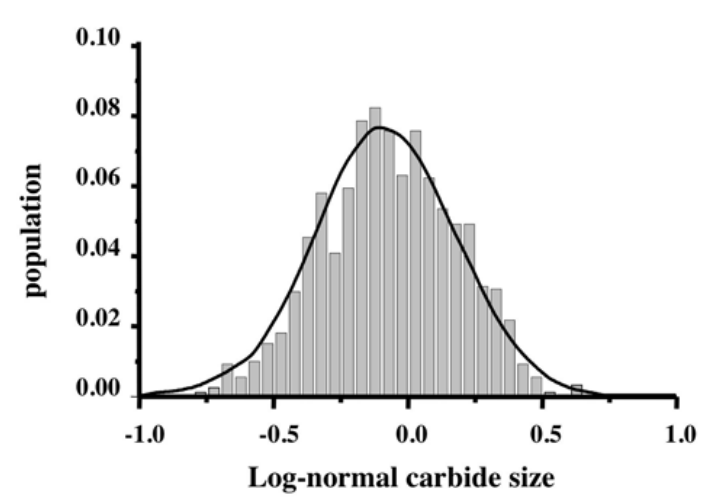

(a)

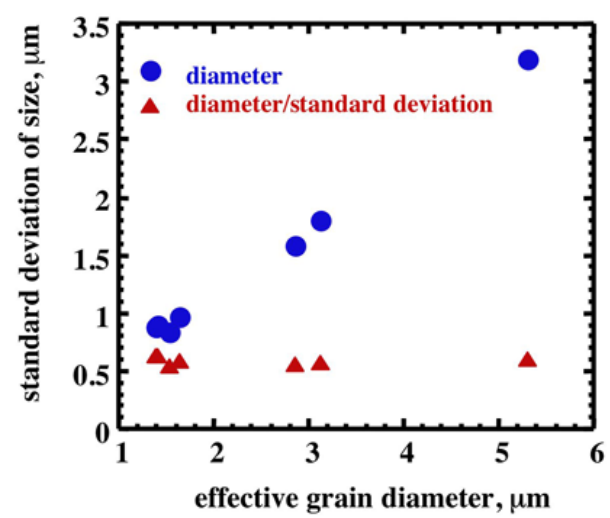

(b)

Figure 3. (a) The carbide grain size distribution of a WC/Co sample with a carbide volume fraction of 0.83 . (b) The relationship between the mean grain diameter and standard deviation of the distribution of grain sizes for a range specimens with different volume fractions and different grain sizes. (Kim et al., 2008)

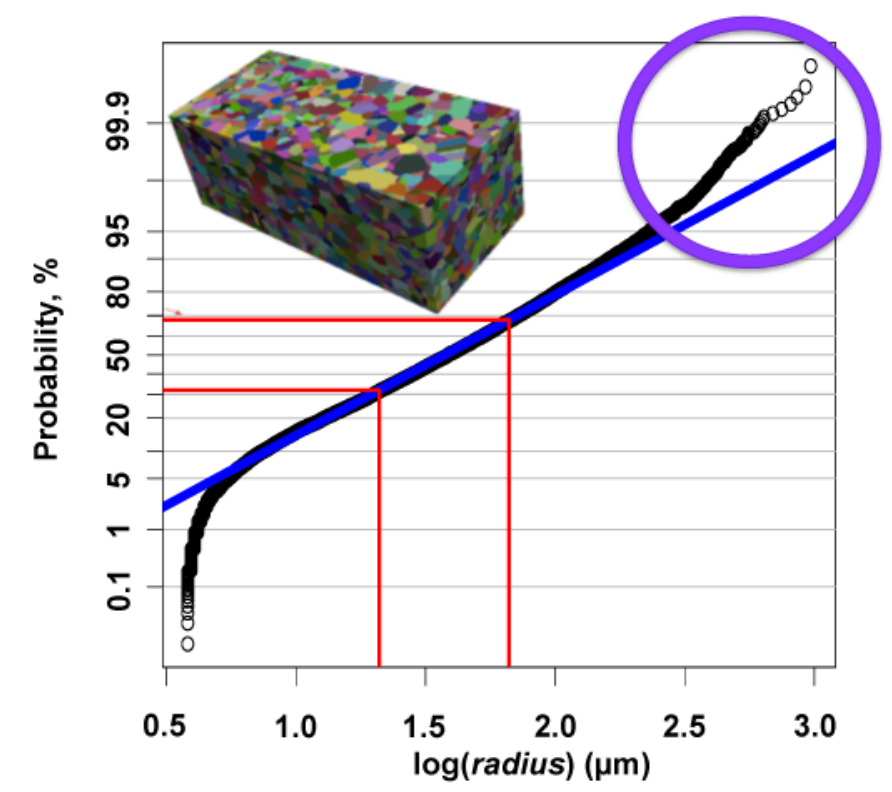

Figure 4. Distribution of $\sim 5800$ grain sizes measured in three dimensions compared to a lognormal distribution (blue line). Red lines are drawn one standard deviation about the mean. The largest grains deviate from this distribution (purple circle). (Tucker et al., 2012) 


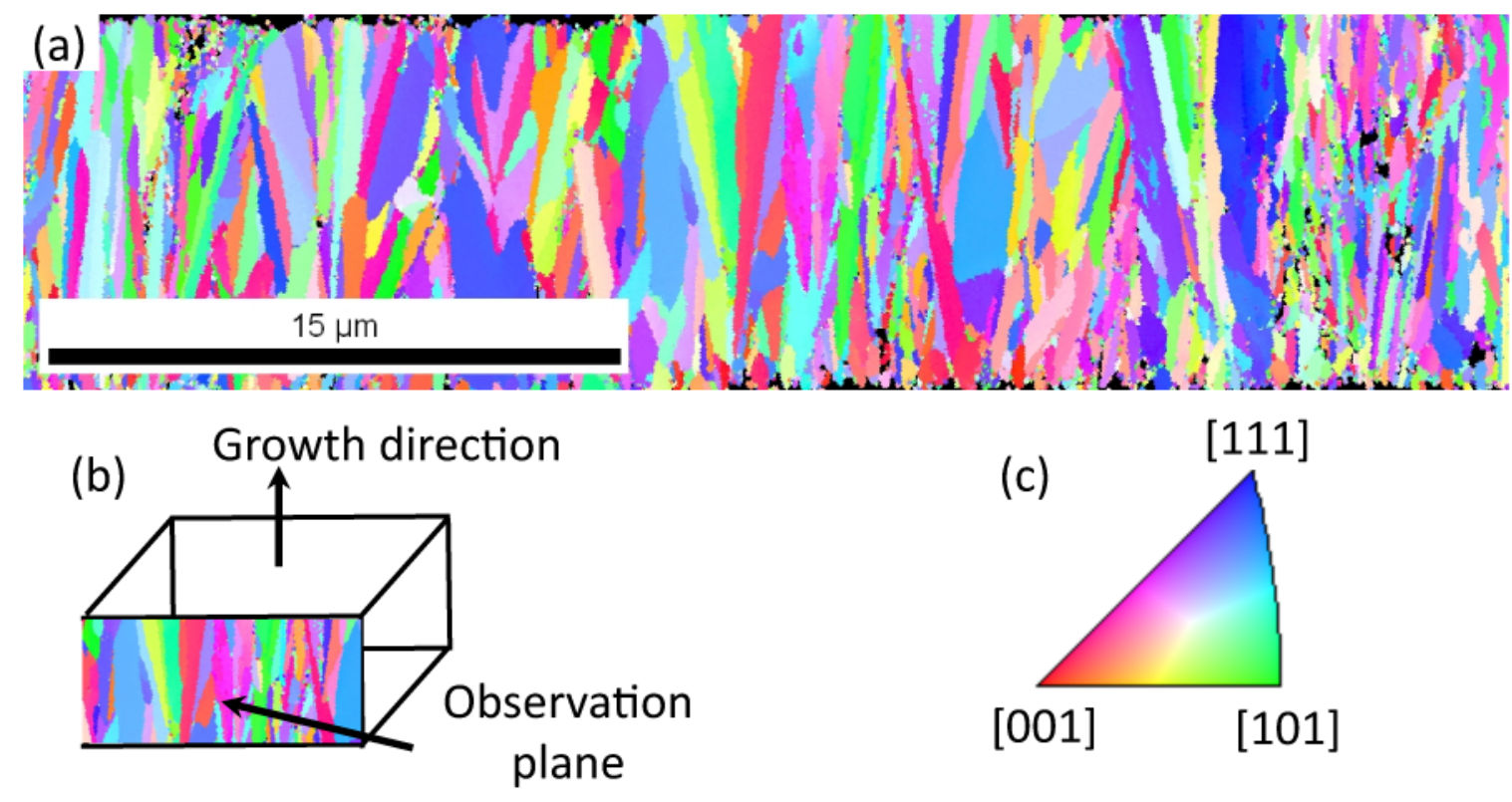

Figure 5. (a) Electron backscatter diffraction orientation map for a $\mathrm{TiC}_{\mathrm{x}} \mathrm{N}_{1-\mathrm{x}}$ coating grown by chemical vapor deposition. (b) This is a transverse section of the coating, as indicated in this schematic. (c) Each color corresponds to a crystal orientation, defined by this key.(Chien et al., 2009) 

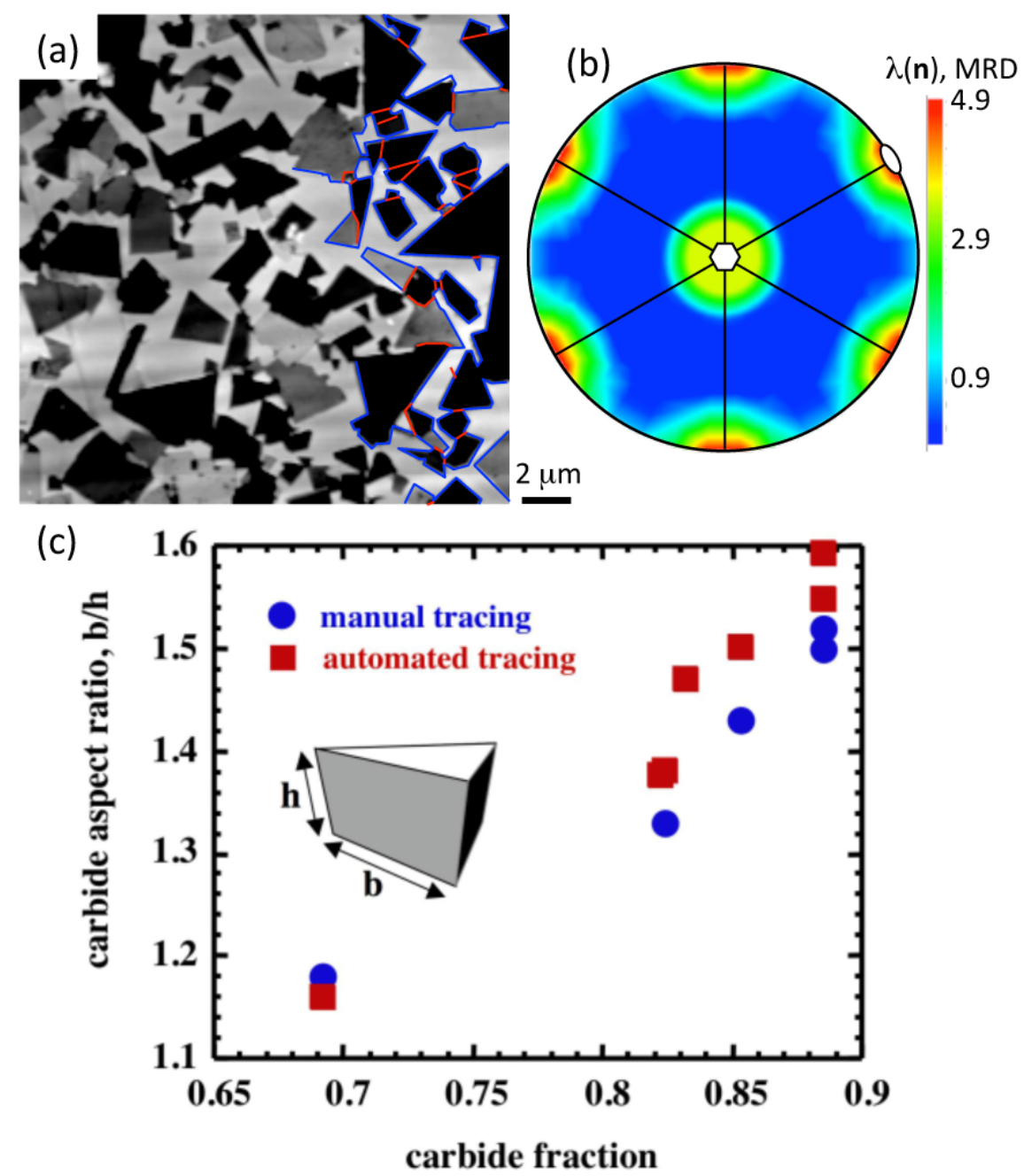

Figure 6. (a) An atomic force microscope image of a WC/Co composite after etching. The dark polygonal features are WC grains. (b) Interface plane distribution for the WC/Co interfaces in a WC/Co composite, showing that particles are dominantly bound by basal and prismatic surfaces. (c) Average base-to-height aspect ratios $(\mathrm{b} / \mathrm{h})$ for the carbide grains in the seven samples, plotted as a function of carbide volume fraction. (Kim et al., 2008) 


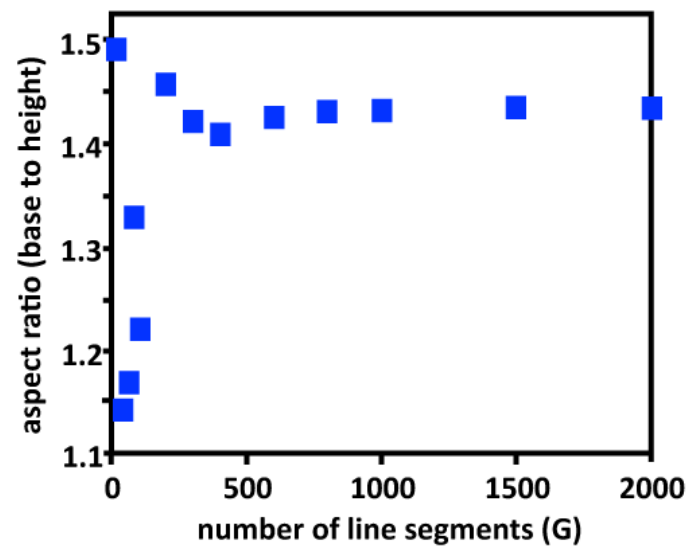

Figure 7. The base-to-height aspect ratio of WC crystals in a WC/Co sample with a carbide volume fraction of 0.85 , determined using different numbers of observations. The result converges after 500 line segments. (Kim et al., 2008)

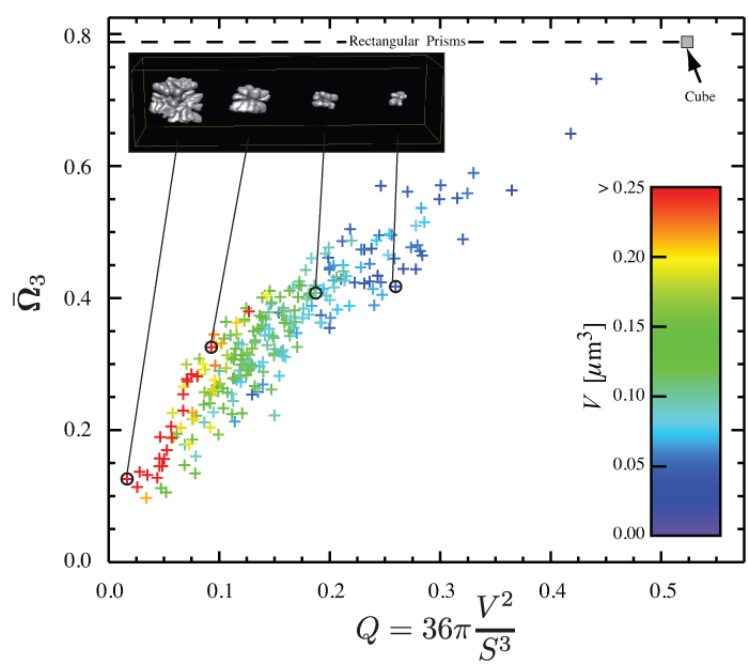

Figure 8. Plot of a moment invariant, $\bar{\Omega}_{3}$, as a function of a shape parameter, $\mathrm{Q}$, for the $260 \gamma^{\prime}$ precipitates from a Rene' 88 -DT superalloy. The color scheme corresponds to the particle volume. The precipitates shown in the inset correspond to the black circles. The horizontal dashed line indicates the location of the class of rectangular prisms, with the cube located at the position indicated on the far right. From (MacSleyne et al., 2009). Copyright Elsevier, reproduced with permission. 


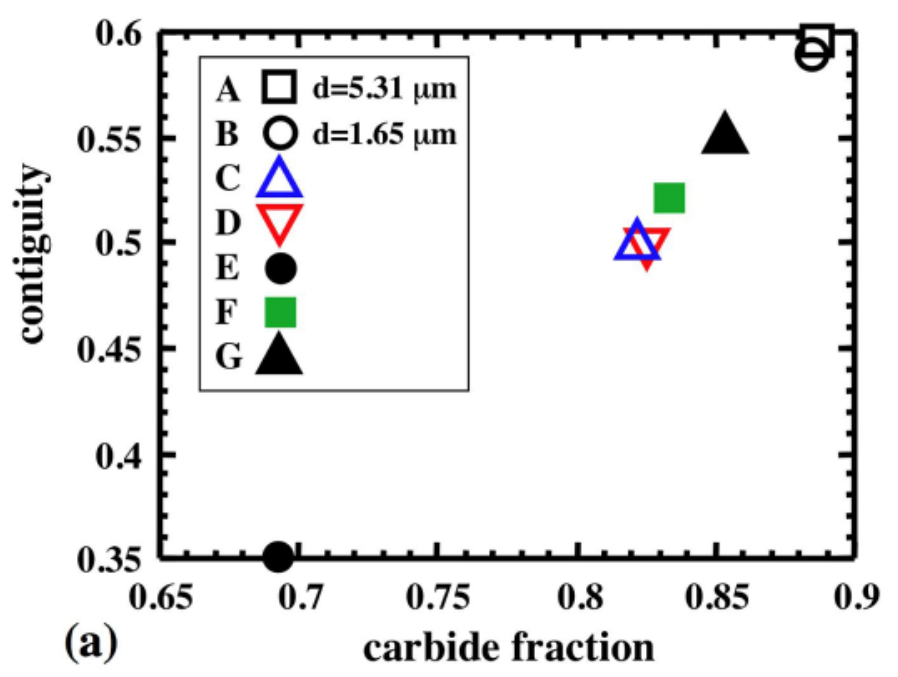

Figure 9. The WC contiguity as a function of the carbide volume fraction for seven different WC/Co specimens. (Kim et al., 2008)

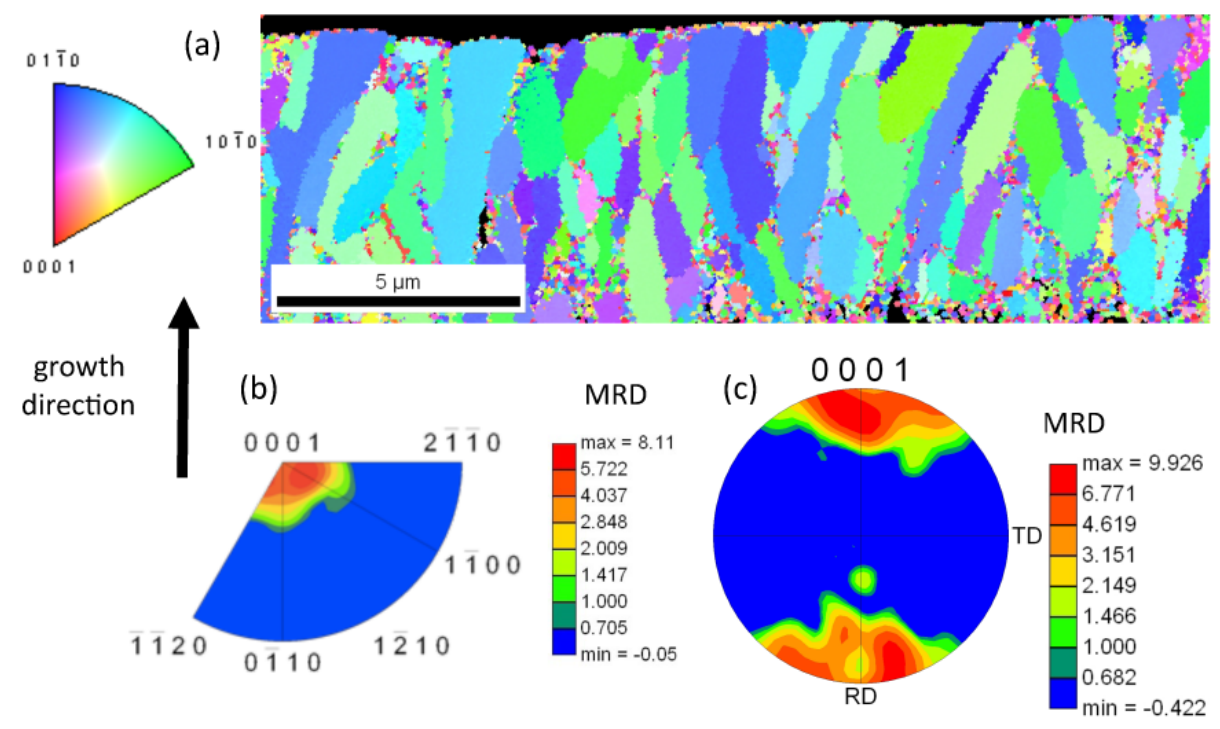

Figure 10. (a) Electron backscatter diffraction orientation map of a transverse section of an $\alpha-\mathrm{Al}_{2} \mathrm{O}_{3}$ coating grown by chemical vapor deposition (color legend for the orientation is shown on the left). (b) Inverse pole figure for the growth direction of the sample. (c) Pole figure for the [0001] directions in the crystal. (Chien et al., 2009) 

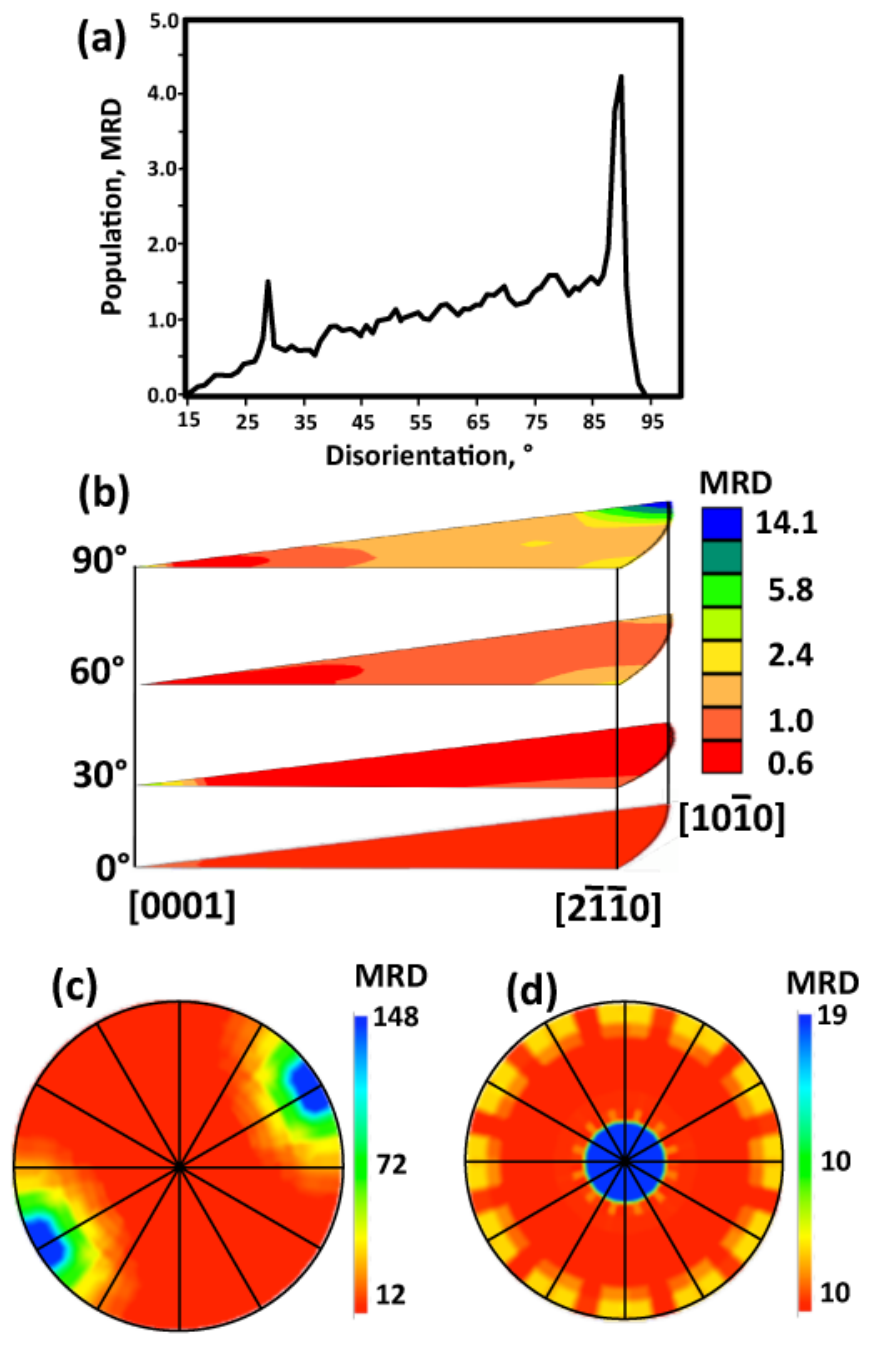

Figure 11. Three different representations of the distribution of relative areas of different types of grain boundaries. (a) Each boundary is classified by its minimum misorientation angle, without consideration of the misorientation axis. (b) Each position in this three-dimensional axis-angle space corresponds to a different misorientation. For any particular axis-angle combination, there is a distribution of grain boundary planes and the distributions for the two peaks in (b) are shown in (c) and (d). (Rohrer, 2011b) 

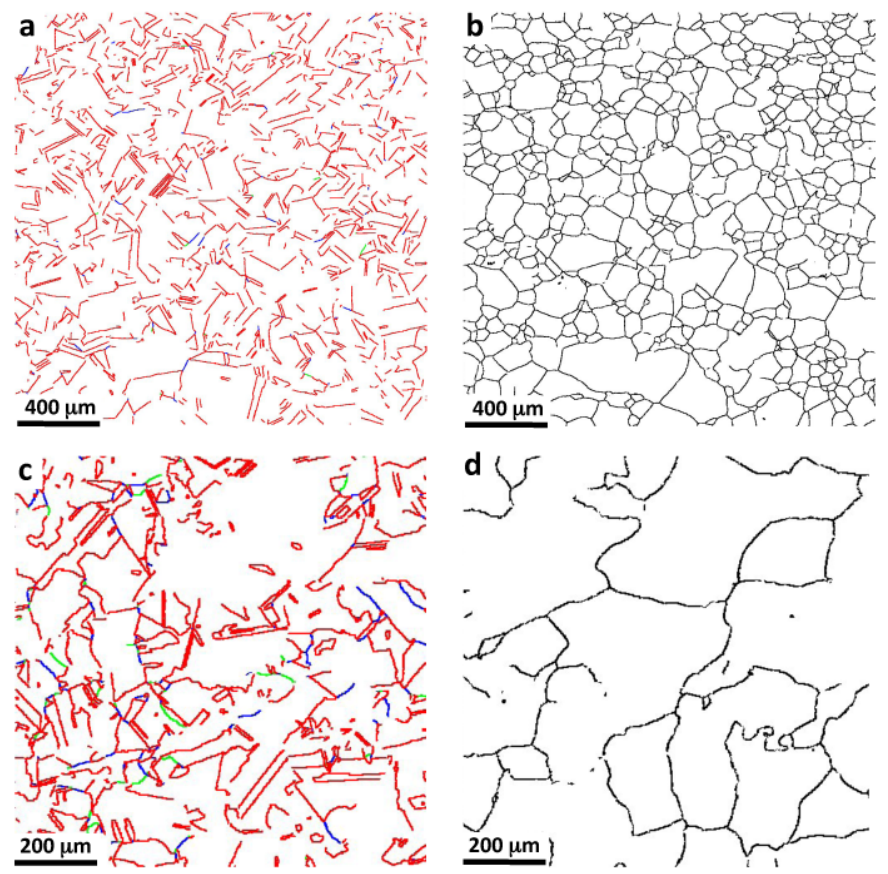

Figure 12. Grain boundary maps for the Ni samples. (a) All of the $\Sigma 3^{\mathrm{n}}$ boundaries in the low $\Sigma 3 \mathrm{Ni}, \beta_{01}=3.1$, (b) all of the non $\Sigma 3^{\mathrm{n}}$ boundaries in the low $\Sigma 3 \mathrm{Ni}, \beta_{01}=$ 0.12 , (c) all of the $\Sigma 3^{\mathrm{n}}$ boundaries in high $\Sigma 3 \mathrm{Ni}, \beta_{01}=0.53$, (d) All of the non- $\Sigma 3^{\mathrm{n}}$ boundaries in high $\Sigma 3 \mathrm{Ni}, \beta_{01}=8.6$. In (a) and (c) the $\Sigma 3$ boundaries are colored red, the $\Sigma 9$ boundaries are blue and the $\Sigma 27$ boundaries are green. (Rohrer and Miller, 2010) 
(a)

(b)

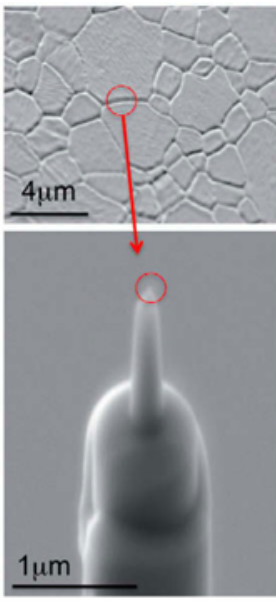

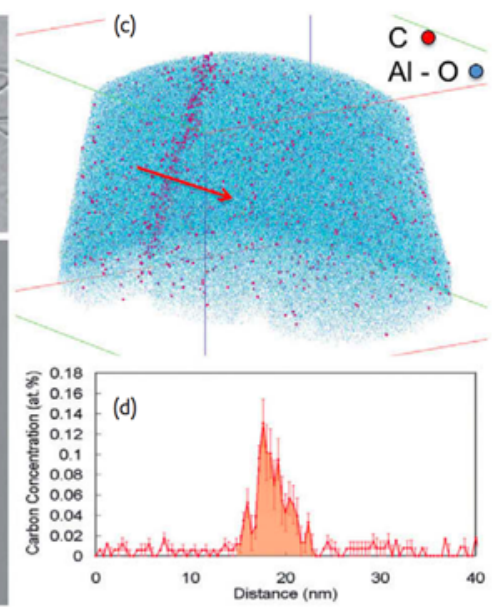

Figure 13. 3DAP analysis of a grain boundary in carbon-doped alumina. (a) SEM image of the etched surface revealing grain boundaries. (b) FIB prepared 3DAP specimen containing a grain boundary near the apex of the specimen; (c) atomprobe reconstruction showing carbon segregation at the grain boundary; (d) Concentration profile across grain boundary along the red arrow shown in b). From (Marquis et al., 2010). Copyright Elsevier, reproduced with permission. 


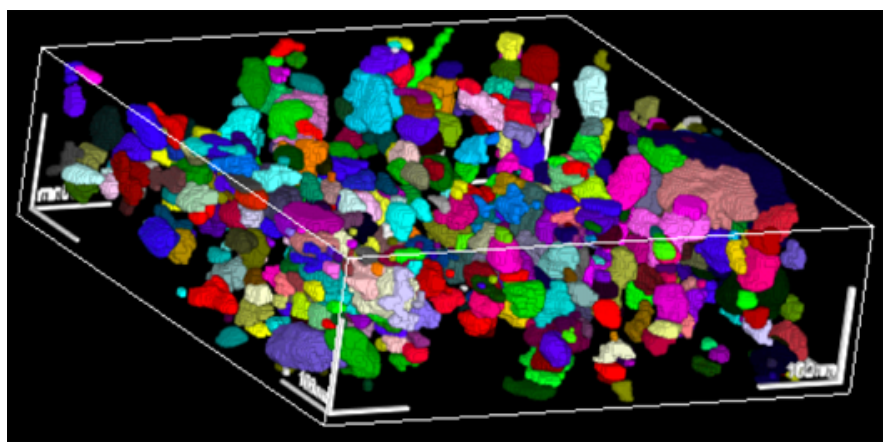

Figure 14. Volume rendered view of the reconstructed mass density distribution of silica inclusions in a rubber matrix. The frame is shown in reconstructed perspective geometry (length and width: $630 \mathrm{~nm}$, thickness: $181 \mathrm{~nm}$ ). The bar for each direction shows the distance of $100 \mathrm{~nm}$. From (Ikeda et al., 2004). Copyright John Wiley and Sons, reproduced with permission.

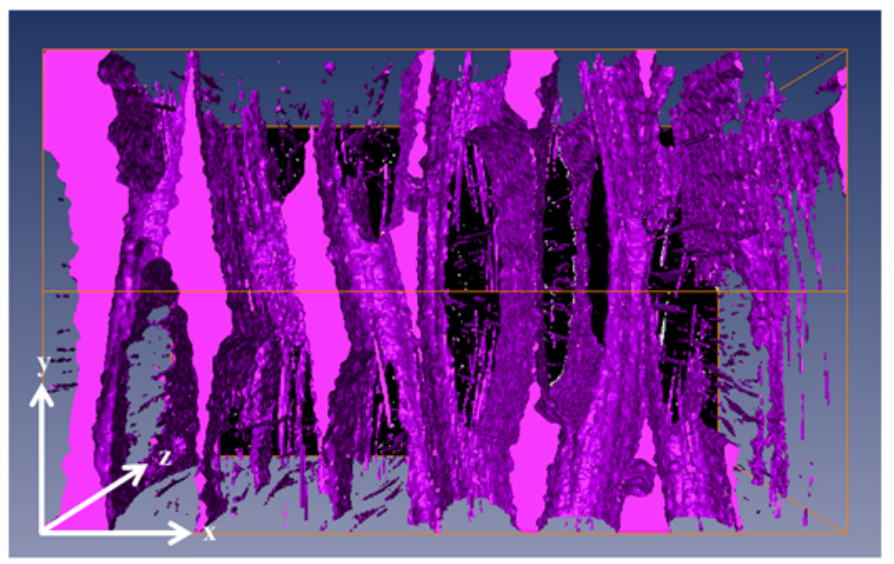

Figure 15. Three-dimensional volume rendering of the axial view of pores in twodimensional $\mathrm{SiC}_{\mathrm{f}}-\mathrm{SiC}$ composites. The dimensions of the reconstructed threedimensional volume is $\mathrm{x} \sim 2.5 \mathrm{~mm}, \mathrm{y} \sim 1.5 \mathrm{~mm}$ and $\mathrm{z} \sim 3.8 \mathrm{~mm}$ (total volume $\sim 14.3$ $\mathrm{mm}^{3}$ ). From (Morales-Rodriguez et al., 2009). Copyright Elsevier, reproduced with permission. 

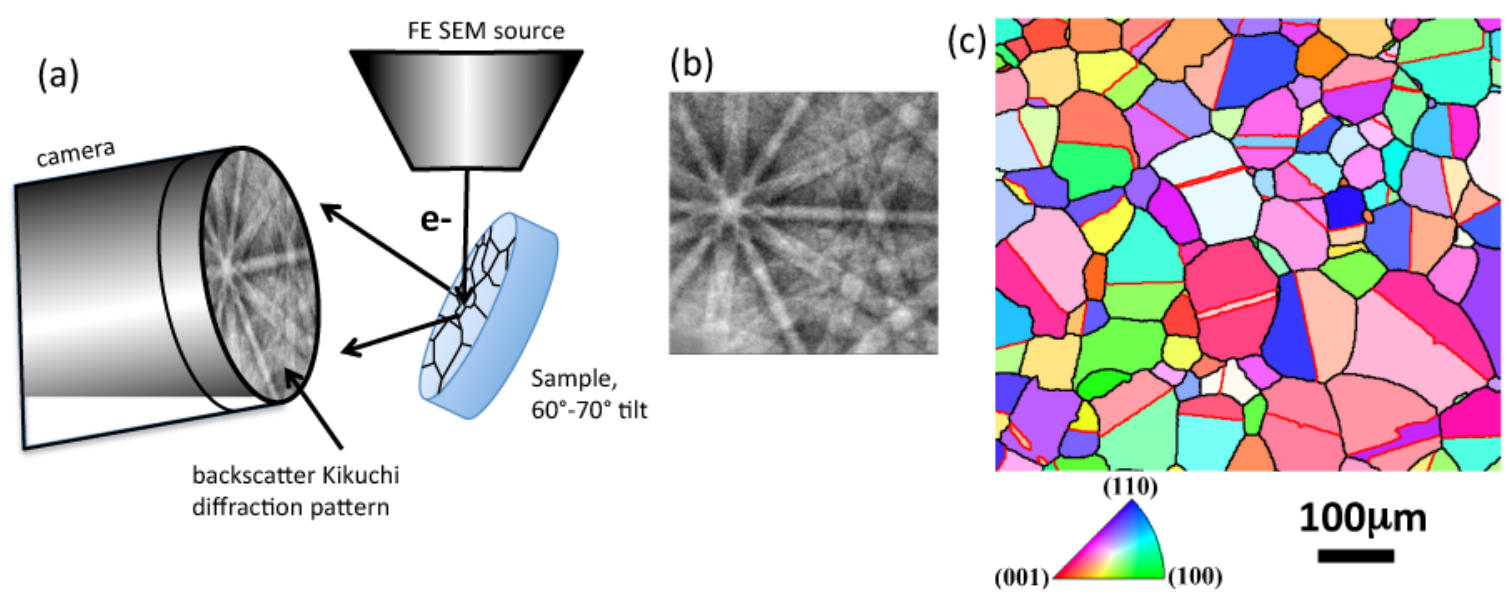

Figure 16. (a) Electron backscatter diffraction (EBSD) maps are recorded in SEM with the sample tilted at a large angle with respect to the beam. (b) A digital camera captures a diffraction pattern that is characteristic of the volume of material that interacted with the beam. (c) After recording local orientations on a grid of points, an orientation map can be plotted. The orientations of the $\mathrm{TiO}_{2}$ sample are specified by colors defined in the legend. (Rohrer, 2011b) 
(a)

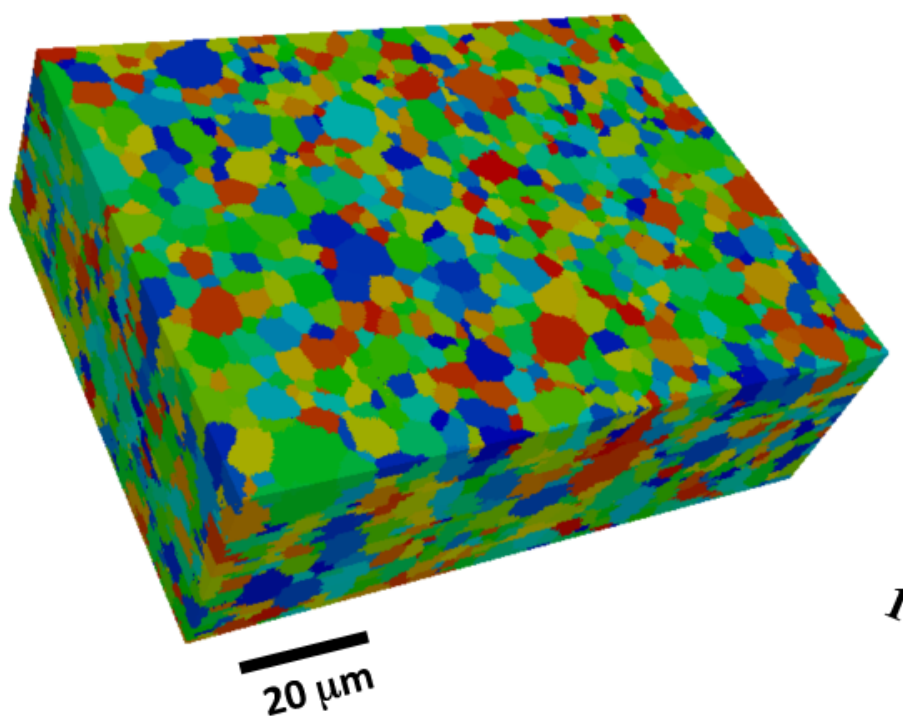

(b)

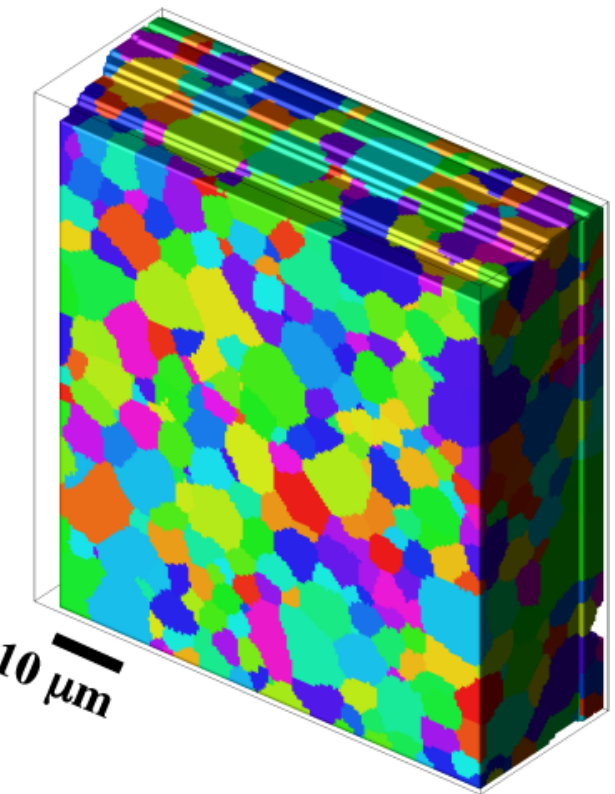

Figure 17. (a) A three-dimensional orientation map of yttria $\left(\mathrm{Y}_{2} \mathrm{O}_{3}\right)$ based on 43 parallel EBSD maps. (Dillon and Rohrer, 2009a) (b) A three-dimensional orientation map of $8 \%$ yttria $\left(\mathrm{Y}_{2} \mathrm{O}_{3}\right)$ stabilized Zirconia $\left(\mathrm{ZrO}_{2}\right)$. (Helmick et al., 2011) 


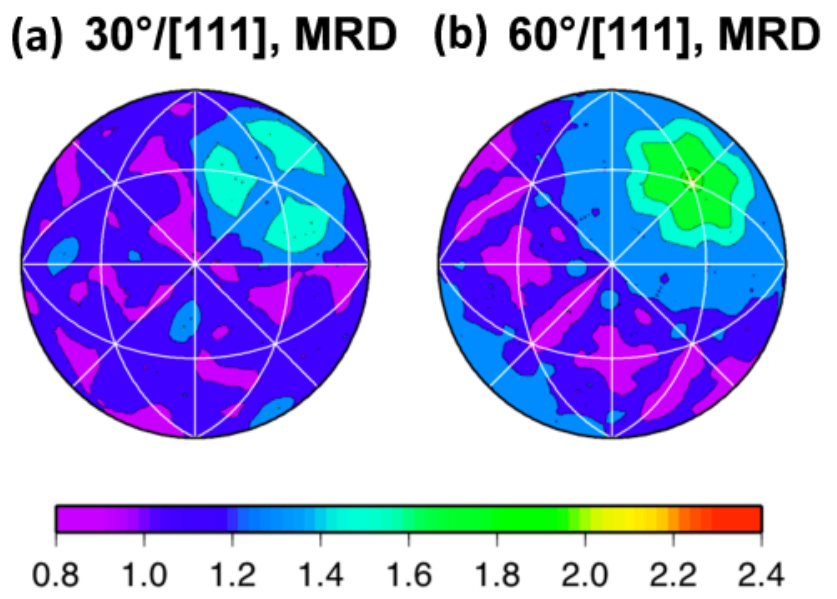

$\begin{array}{ll}\text { (c) } 30 \%[111] \text {, a.u. } & \text { (d) } 60 \%[111] \text {, a.u. }\end{array}$
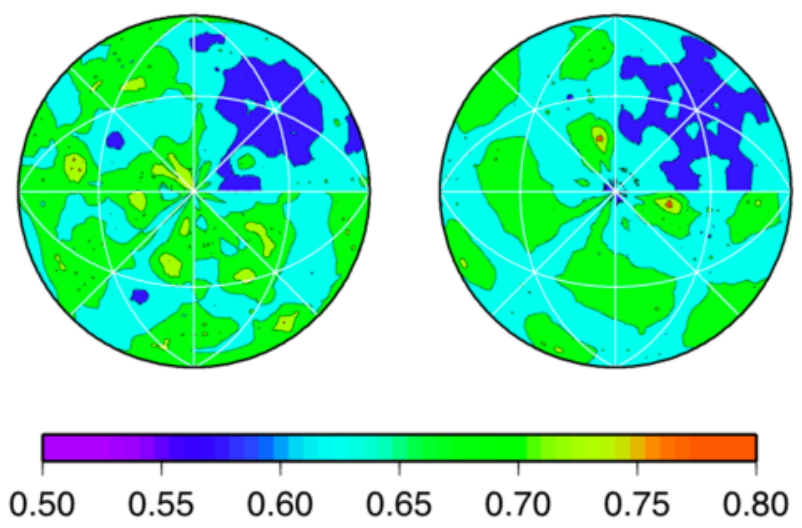

Figure 18. (a-b) The grain-boundary plane distribution at the fixed misorientations of $30^{\circ} /[111]$ and $60^{\circ} /[111]$, plotted in stereographic projection along [001]. The corresponding energy distributions are shown in (c) and (d). (Dillon and Rohrer, 2009a) 

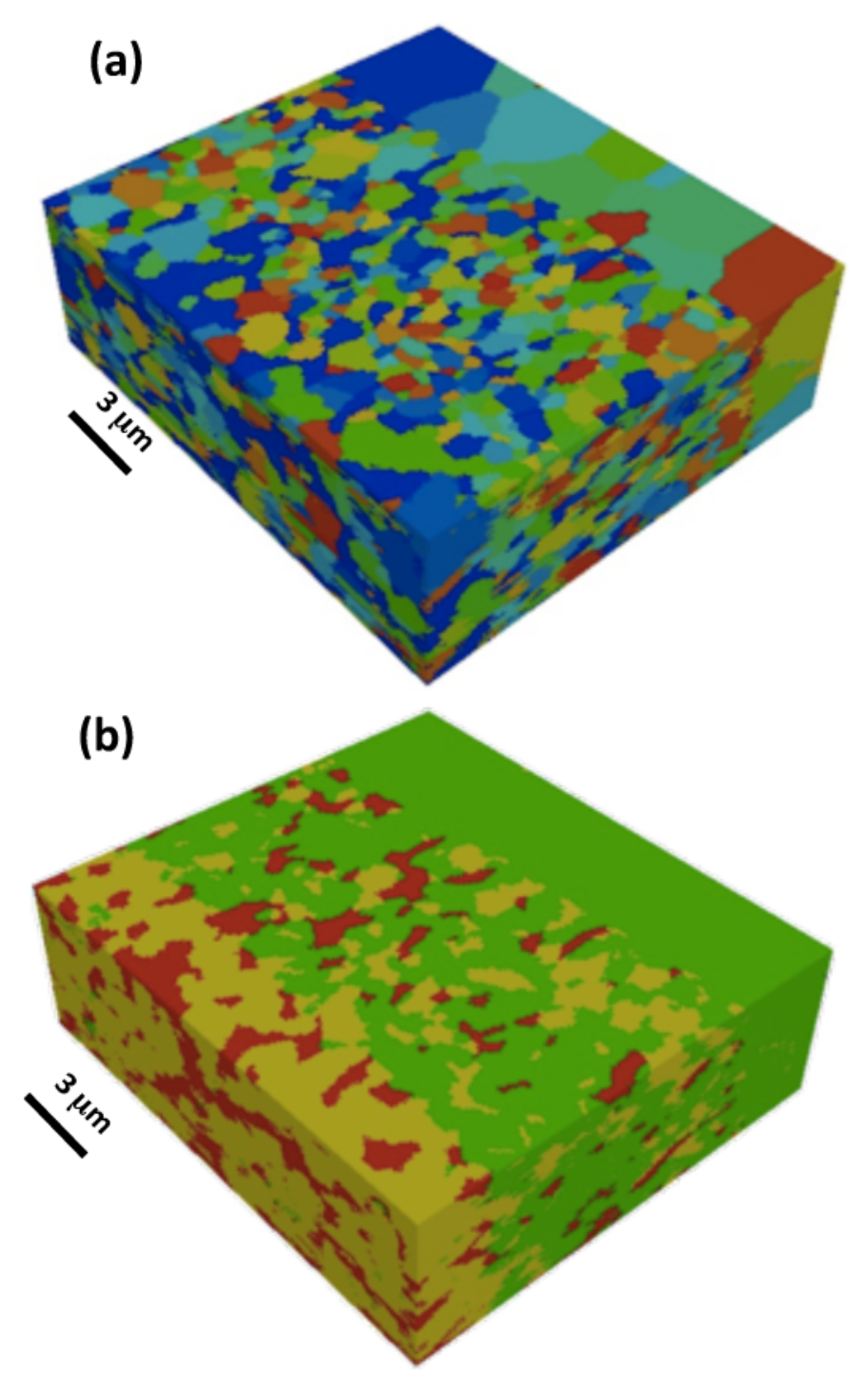

Figure 19. Oblique projections of the reconstructed three-dimensional volume of the active cathode of a solid oxide fuel cell. In the orientation map in (a), different colors are used to indicate different grains. In the phase map (b), green indicates YSZ, gold indicates LSM, and red indicates pores. (Helmick et al., 2011) 

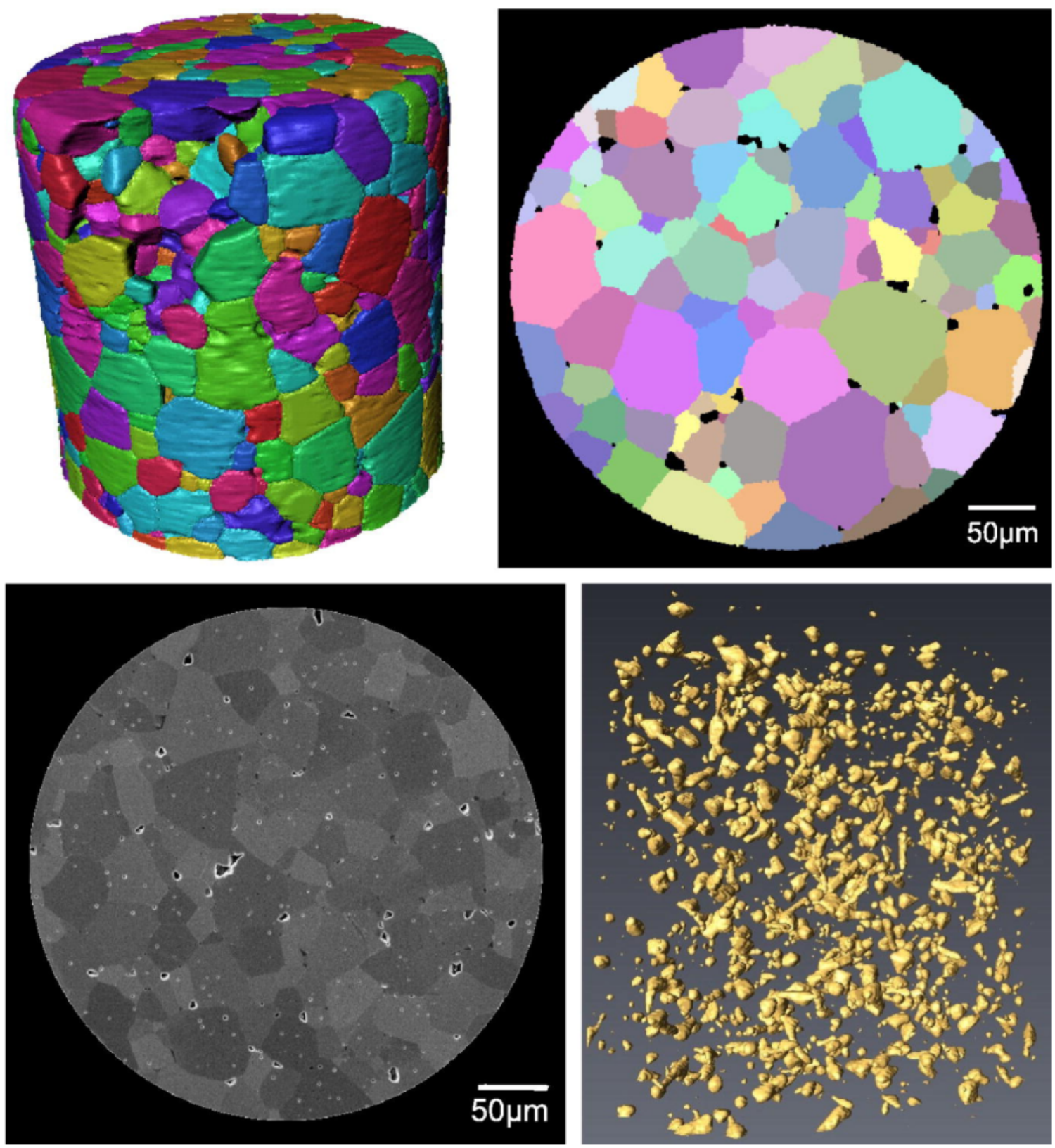

Figure 20. Three-dimension view of a $\mathrm{SrTiO}_{3}$ polycrystal (a) (colored randomly) and cross-section (colored according to crystallographic orientation) (b) of the reconstructed structure compared to a SEM micrograph (c) of the same material. Collective ensemble of intergranular pores (d). (Syha et al., 2012) Copyright Elsevier, reproduced with permission. 